CLINICAL REVIEW

\title{
Daytime sleepiness versus fatigue in patients with multiple sclerosis: A systematic review on the Epworth sleepiness scale as an assessment tool
}

\author{
Roland F.J. Popp a , Anna K. Fierlbeck a , Helge Knüttel ${ }^{\text {b }}$, Nicole König ${ }^{\text {c }}$, Rainer Rupprecht ${ }^{\text {a }}$, \\ Robert Weissert $^{\mathrm{c}, 1}$, Thomas C. Wetter ${ }^{\text {a, }}{ }^{*, 1}$ \\ a Department of Psychiatry and Psychotherapy, Center of Sleep Medicine, University of Regensburg, Germany \\ b University Library of Regensburg, University of Regensburg, Germany \\ ${ }^{\mathrm{c}}$ Department of Neurology, University of Regensburg, Germany
}

\section{A R T I C L E I N F O}

\section{Article history:}

Received 26 August 2015

Received in revised form 26 February 2016

Accepted 9 March 2016

Available online $\mathrm{xxx}$

\section{Keywords:}

Multiple sclerosis

Fatigue

Daytime sleepiness

Epworth sleepiness scale

Systematic review

\begin{abstract}
S U M M A R Y
Fatigue is a frequent and distressing symptom in patients with multiple sclerosis (MS). In contrast, sleepiness, characterized by difficulties to stay awake and alert during the day, seems to be less prevalent in MS; however, exact studies are lacking. In addition, there is a semantic confusion of the concepts of "fatigue" and "sleepiness", which are often used interchangeably. We conducted a systematic review of studies using the Epworth sleepiness scale (ESS) for the assessment of daytime sleepiness in patients with MS. The summarized results of 48 studies demonstrate that sleepiness, as indicated by elevated ESS scores, is less prevalent and less severe than fatigue but is present in a significant proportion of patients with MS. In most cross-sectional and longitudinal studies, there was a moderate association between ESS scores and various fatigue rating scales. Longitudinal studies on the effect of wakefulness-promoting agents failed to show a consistent improvement of sleepiness or fatigue as compared to placebo. It has also been shown that daytime sleepiness is frequently associated with comorbid sleep disorders that are often underrecognized and undertreated in MS. Sleepiness and potential sleep disorders may also precipitate and perpetuate fatigue in patients with MS and should be part of the differential diagnostic assessment. To support an appropriate decision-making process, we propose a stepwise evaluation of sleepiness as compared to fatigue in patients with MS.
\end{abstract}

๑) 2016 Elsevier Ltd. All rights reserved.

\section{Introduction}

Multiple sclerosis (MS) is an inflammatory, demyelinating and neurodegenerative autoimmune disease of the central nervous system (CNS) [1]. It is the leading cause of non-traumatic neurologic disability in young adults [2]. MS is a chronic disease, whose clinical course can be defined as relapsing-remitting, primaryprogressive and secondary-progressive, and progressive-relapsing (http://www.nationalmssociety.org) [3]. It involves a spectrum of neurologic symptoms, such as sensory disturbances, impaired vision, paresis, gait difficulties and bladder dysfunction. In addition, fatigue, as well as cognitive decline, reflects the presence and

\footnotetext{
* Corresponding author. Department of Psychiatry and Psychotherapy, Center of Sleep Medicine, University of Regensburg, Universitätsstrasse 84, D-93053, Regensburg, Germany. Tel.: +49 (0) 941941 5068; fax: +49 (0) 9419411005 E-mail address: thomas.wetter@medbo.de (T.C. Wetter).

1 The last two authors contributed equally to this study.
}

distribution of damage in the CNS and may vary considerably among individuals. MS-related fatigue is ascribed to multifactorial etiologies including inflammatory cytokines, nocturia, pain, infection, anxiety and depression [4]. In addition, poor sleep and sleep disorders, such as restless legs syndrome (RLS), have been identified as contributing factors for MS-related fatigue and are more common in MS patients compared to healthy controls $[5,6]$.

It is reported that 53-92\% of patients with MS are affected by fatigue and as many as $46-66 \%$ suffer on a daily basis [7-12]. Fatigue is a disabling symptom that can be described as a feeling of tiredness, exhaustion, weariness or lassitude. It is commonly measured by self-rating scales such as the fatigue severity scale (FSS) [13]. In about one third of the patients, fatigue may present as the initial symptom of MS [14]. Overall, 28-60\% of patients report that fatigue is their most distressing symptom [12,14,15], being a major cause of unemployment and early retirement in MS $[16,17]$. Severe fatigue is also related to increased instances of physical disability, neurological impairment and mobility 


\section{List of abbreviations}

CBT cognitive behavioral therapy

CPAP continuous positive airway pressure

ESS Epworth sleepiness scale

FIS fatigue impact scale

FSMC fatigue scale for motor and cognitive functions

FSS fatigue severity scale

ICSD-3 International classification of sleep disorders (third edition)

IFN interferon

MFIS modified fatigue impact scale

MS multiple sclerosis

NFI-MS neurological fatigue index

PLMD periodic limb movement disorder

PSG polysomnography

PSQI Pittsburgh sleep quality index

RLS restless legs syndrome

SRBD sleep-related breathing disorder impairment (e.g., bedridden patients reported the most severe fatigue) [16].

Fatigue is distinct from sleepiness, which is defined by the International classification of sleep disorders (ICSD-3) as the inability to stay awake and alert during the day, leading to episodes of an irrepressible need for sleep or unintended lapses into drowsiness or sleep [18] (Table 1). Sleepiness predisposes people to develop serious performance impairments in daily functioning and is a risk factor for potentially life-threatening domestic, occupational, and vehicular accidents [18]. The complaint of excessive sleepiness during the normal wake period is also a pivotal symptom for sleep disorders of hypersomnolence, as classified by the ICSD-3.

Recently, sleep disorders in MS, as well as the causes and consequences of daytime sleepiness in patients with MS, have gained more attention in research, suggesting that sleepiness is an underrecognized and overlooked symptom in MS. In contrast to the huge body of literature on the presence of fatigue in MS, to our knowledge, there is no systematic epidemiological study on the frequency of sleepiness in MS.

The wide spectrum of fatigue prevalence rates in MS patients may be due to the use of different assessment methods based on various definitions and interpretations of the term "fatigue". In clinical practice as well as in the scientific literature, "fatigue" and "sleepiness" are frequently used interchangeably [19]. Individuals

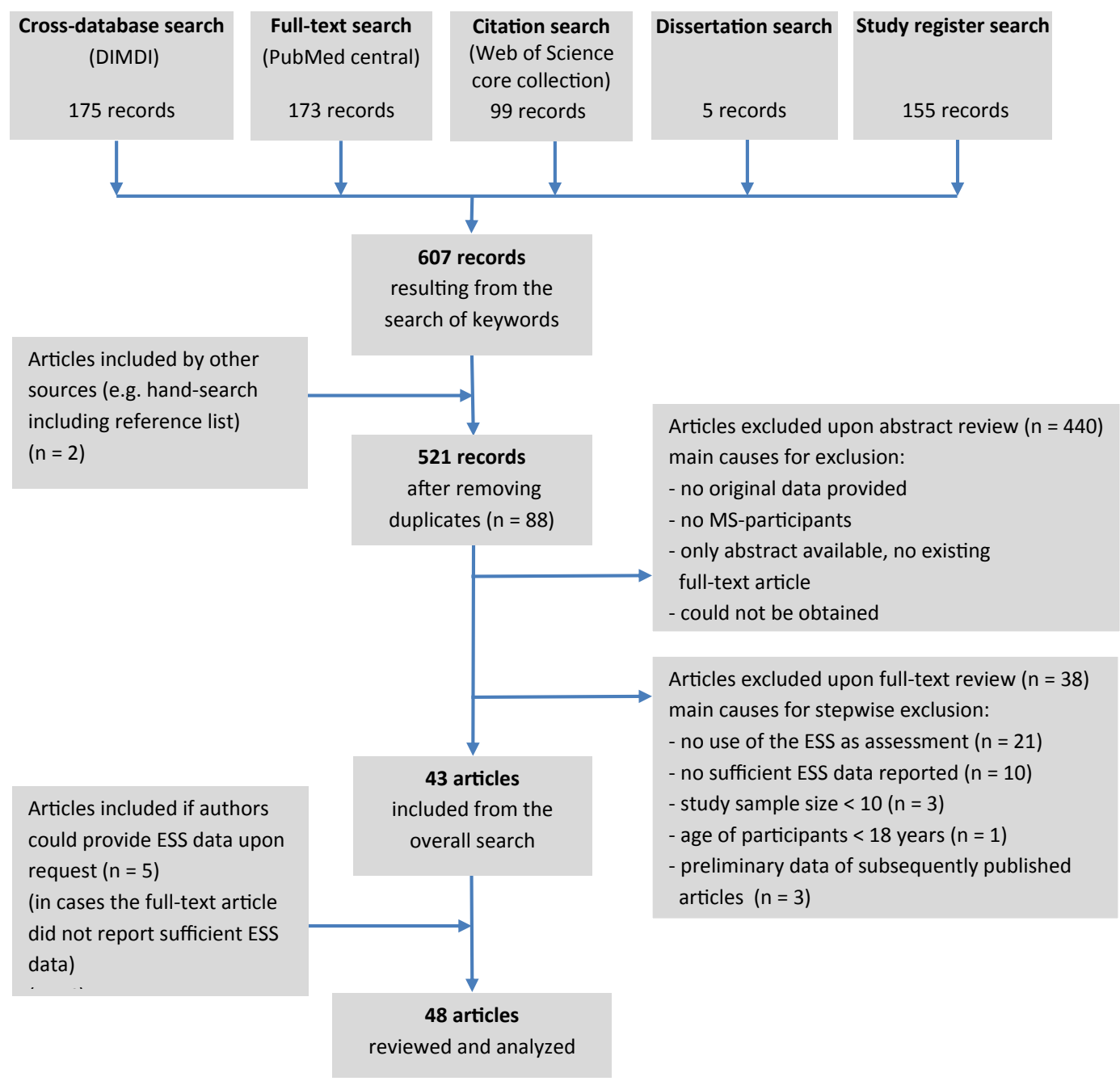

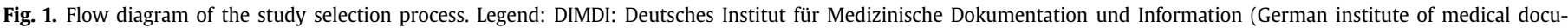
mentation and information); MS: multiple sclerosis; ESS: Epworth sleepiness scale. 
Table 1

Comparison of fatigue and sleepiness as two different domains.

\begin{tabular}{|c|c|c|}
\hline & Fatigue & Sleepiness \\
\hline Definition & $\begin{array}{l}\text { "Fatigue is a subjective lack of physical and/or mental energy } \\
\text { that is perceived by the individual or the caregiver to interfere } \\
\text { with usual and desired activities." (p. 2) [82]. }\end{array}$ & $\begin{array}{l}\text { "Sleepiness is the inability to stay awake and alert } \\
\text { during the major waking episodes of the day, } \\
\text { resulting in periods of irrepressible need for sleep } \\
\text { or unintended lapses into drowsiness or sleep." (p. } \\
\text { 143) [18]. }\end{array}$ \\
\hline Symptoms & $\begin{array}{l}\text { - Feelings of tiredness, exhaustion, weariness or lassitude. } \\
\text { - Not necessarily associated with sleep pressure. } \\
\text { - No definite sleep drive when resting (e.g., lying down to } \\
\text { relax). }\end{array}$ & $\begin{array}{l}\text { - Decreased level of alertness or wakefulness. } \\
\text { - Increased tendency to fall asleep or doze off } \\
\text { unintentionally. } \\
\text { - Sleep drive when resting (e.g., lying down to } \\
\text { nap). }\end{array}$ \\
\hline Semantics & $\begin{array}{l}\text { - Tiredness is commonly used as synonym (e.g., by } \\
\text { insomniacs). }\end{array}$ & $\begin{array}{l}\text { - Tiredness is commonly used as synonym (e.g., } \\
\text { by narcoleptics). }\end{array}$ \\
\hline Characteristics in MS & $\begin{array}{l}\text { - Common and most troublesome symptom. } \\
\text { - Strong negative impact on social and occupational } \\
\text { functioning. }\end{array}$ & $\begin{array}{l}\text { - Not commonly associated with MS. } \\
\text { - Often associated with sleep disorders. }\end{array}$ \\
\hline Assessment by common rating scales & $\begin{array}{l}\text { Fatigue severity scale (FSS) } \\
\text { - Most widely used validated scale. } \\
\text { - Evaluates the impact on motivation, physical abilities and } \\
\text { social functioning. } \\
\text { - Self-administered, nine items. } \\
\text { - } 7 \text {-point Likert scale from } 1 \text { (strongly disagree) to } 7 \\
\text { (strongly agree). } \\
\text { - Critical cut-off: mean FSS score }>4 \text { (min: } 1 \text {; max: } 7 \text { ) or } \\
\text { total FSS score } \geq 36 \text { (min: } 9 \text {; max: } 63 \text { ). }\end{array}$ & $\begin{array}{l}\text { Epworth sleepiness scale (ESS). } \\
\text { - Most commonly used scale in sleep research } \\
\text { and clinical settings. } \\
\text { - Assessment of sleep propensity } \\
\text { - Self-administered, 8-item questionnaire. } \\
\text { - 4-point Likert scale (from } 0 \text { to } 3 \text { ) to rate the } \\
\text { likelihood of dozing off in eight everyday } \\
\text { situations. } \\
\text { - Critical cut-off: total ESS score }>10 \text { (min: } 0 \text {; } \\
\text { max: } 24 \text { ). }\end{array}$ \\
\hline Objective assessments & - No validated measures available. & $\begin{array}{l}\text { - Maintenance of wakefulness test; multiple } \\
\text { sleep latency test; EEG; pupillography. } \\
\text { - Psychomotor vigilance task; sustained } \\
\text { attention and vigilance tasks; driving } \\
\text { simulator. }\end{array}$ \\
\hline Counter-measures & $\begin{array}{l}\text { - Alleviated by periods of rest, unlike weakness (asthenia). } \\
\text { - Limited efficacy of stimulants. }\end{array}$ & $\begin{array}{l}\text { - Most effective: sleep or short naps, but not } \\
\text { rest. } \\
\text { - Stimulants temporarily effective. }\end{array}$ \\
\hline
\end{tabular}

EEG: electroencephalography; ESS: Epworth sleepiness scale; FSS: fatigue severity scale; MS: multiple sclerosis.

may even subsume both terms under the complaint of "being tired". Nevertheless, "fatigue", "sleepiness" or "tiredness" refer to distinct concepts and provide different semantic connotations [20]. The need to distinguish between fatigue and sleepiness is supported by other studies showing that both conditions substantially differ in implications for diagnosis and treatment, subjective experience, and their underlying neurobiological mechanisms [19,21,22].

A widely used approach to evaluate daytime sleepiness on a subjective level is the employment of the Epworth sleepiness scale (ESS), a self-administered 8-item questionnaire assessing sleep propensity [23]. A total ESS score greater than 10 (range 0-24) is indicative for increased sleepiness. In the instructions, the ESS asks for the likelihood of dozing off or falling asleep in different everyday situations, "in contrast to feeling just tired" [23]. Thus, the ESS quantifies daytime sleepiness using behavioral correlates and circumvents subjective evaluations of states of tiredness, sleepiness or fatigue. Assessing sleepiness by this approach avoids semantic confusion, which is a problem when using fatigue questionnaires that are based on self-reports.

The aim of this systematic review is to summarize the results of published studies using the ESS for the assessment of daytime sleepiness in patients with MS. We focused on the frequency and extent of daytime sleepiness compared to fatigue as assessed by self-administered rating scales such as the FSS or other related scales (e.g., neurological fatigue index (NFI-MS), modified fatigue impact scale (MFIS)).

\section{Methods}

Literature search and identification of studies

Studies were identified by searching electronic databases and scanning reference lists of articles. The present review only includes studies that used the ESS, which was published in 1991, as an assessment-tool. Therefore, articles that were published before 1991 were not considered. No other limits (e.g., language restrictions) were applied. The search strategy was developed by a subject specialist (RP) in collaboration with an information specialist and librarian who is trained and experienced in conducting comprehensive literature searches (HK). Database searches were conducted by HK on May 14, 2014, with an update on April 22, 2015. In addition, registers for clinical trials were searched on August 04, 2015. While our search strategy was not peer-reviewed, we strived to design, carry out and report the literature search according to current checklists and recommendations [24,25].

The research question was translated into two search concepts, "multiple sclerosis" and "Epworth sleepiness scale" that were combined using the Boolean operator AND. The search strategy was adapted to the various databases and search interfaces. This included selecting feasible search terms, syntax, and relevant subject headings. The searches were designed to be sensitive and potentially over-inclusive to avoid missing any relevant articles. We searched 28 medical and psychological reference databases hosted by DIMDI (Deutsches Institut für Medizinische Dokumentation und Information [German institute of medical documentation and information]), including MEDLINE, Embase, Cochrane database of systematic reviews, and Cochrane central register of controlled trials. In addition, a full text search was conducted in PubMed central. In Web of Science core collection, we searched for studies on MS that cite the original article about the "Epworth sleepiness scale" by Johns, [23]. Several dissertation databases and four registers for clinical trials were also consulted.

A detailed documentation of the searches allowing for replication, can be seen in Appendix 1, which is available as an electronic 


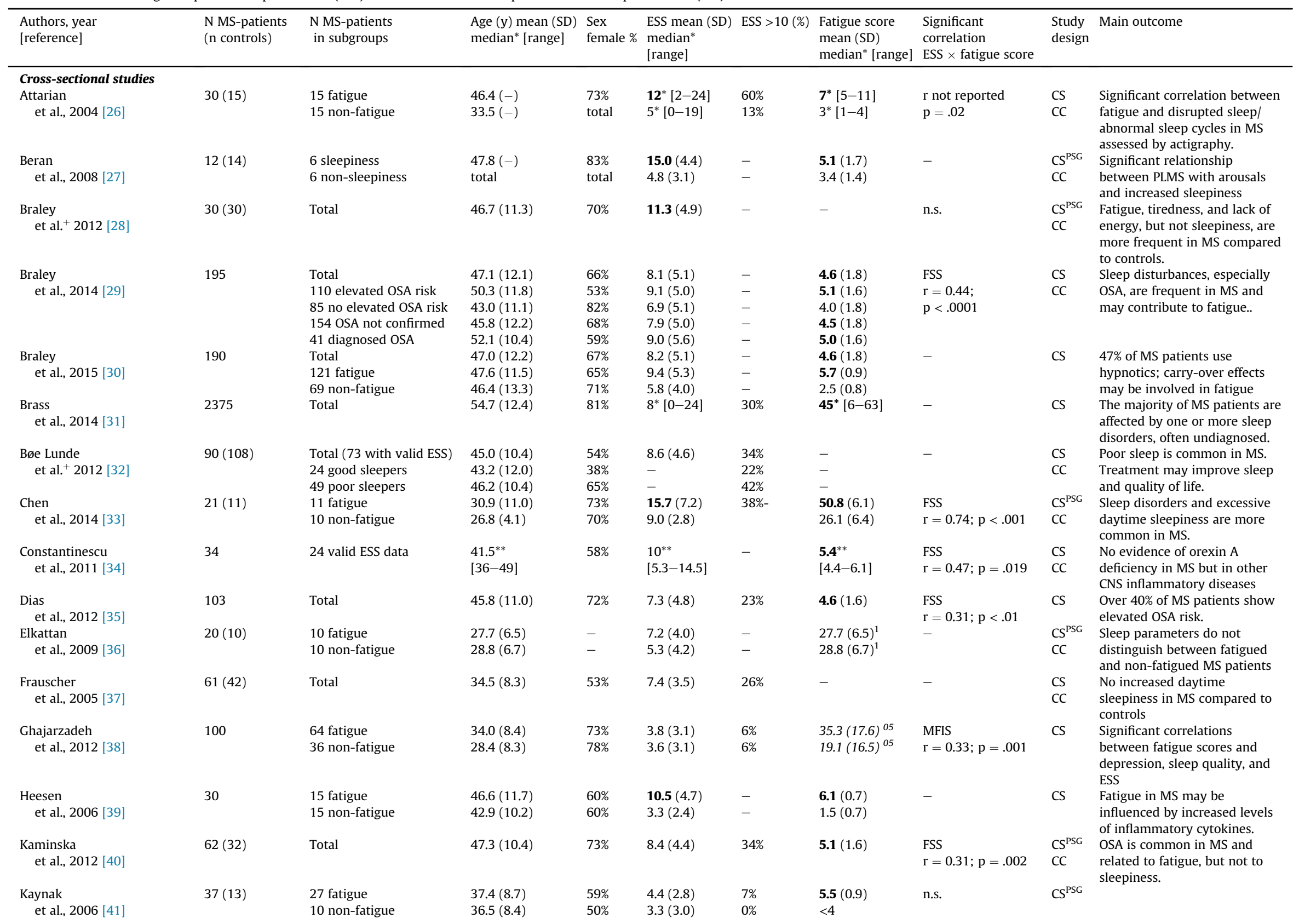




\begin{tabular}{|c|c|c|c|c|c|c|c|c|c|c|}
\hline $\begin{array}{l}\text { Kister } \\
\quad \text { et al., } 2010[42]\end{array}$ & 167 & $\begin{array}{l}94 \text { migraineurs } \\
73 \text { no headache }\end{array}$ & $\begin{array}{l}43(11.0) \\
47(13.0)\end{array}$ & $\begin{array}{l}90 \% \\
57 \%\end{array}$ & $\begin{array}{l}8.1(-) \\
5.6(-)\end{array}$ & $\begin{array}{l}- \\
-\end{array}$ & $\begin{array}{l}\mathbf{5 . 0}(-) \\
3.6(-)\end{array}$ & - & CS & $\begin{array}{l}\text { in MS fatigue. } \\
\text { Migraine in MS is more } \\
\text { frequent than in the general } \\
\text { population. }\end{array}$ \\
\hline $\begin{array}{l}\text { Knudsen } \\
\text { et al., } 2008 \text { [43] }\end{array}$ & $48 \mathrm{MS}$ and MON & Total & [21-57] & $71 \%$ & $5.6(2.9)$ & $10 \%$ & - & - & CS & $\begin{array}{l}\text { Intact hypocretin system in } \\
\text { both subgroups, no increased } \\
\text { sleepiness }\end{array}$ \\
\hline $\begin{array}{l}\text { Kotterba } \\
\quad \text { et al., } 2003[44]\end{array}$ & 31 & Total & $35.6(8.3)$ & $58 \%$ & $6.1(2.9)$ & $10 \%$ & $4.3(1.6)$ & $\begin{array}{l}\mathrm{FSS} \\
\mathrm{r}=0.42 ; \mathrm{p}<.05\end{array}$ & CS & $\begin{array}{l}\text { Terms "sleepiness" and } \\
\text { "fatigue" are often confused, } \\
\text { but different scales allow for } \\
\text { differentiation }\end{array}$ \\
\hline $\begin{array}{l}\text { Labuz-Roszak } \\
\text { et al., } 2012 \text { [45] }\end{array}$ & 122 & Total & $37.7(10.8)$ & $71 \%$ & $6.3(3.9)$ & $21 \%$ & $40.6(14.8)^{1}$ & $\begin{array}{l}\mathrm{FSS} \\
\mathrm{r}=0.18 ; \mathrm{p}=.03\end{array}$ & CS & $\begin{array}{l}\text { Fatigue is frequent in MS and } \\
\text { moderately correlated with } \\
\text { depression and anxiety. }\end{array}$ \\
\hline $\begin{array}{l}\text { Merkelbach } \\
\quad \text { et al., } 2011 \text { [46] }\end{array}$ & 80 & Total & $43.2(9.8)$ & $71 \%$ & $8.1(3.7)$ & - & $4.4(1.6)$ & $\begin{array}{l}\mathrm{FSS} \\
\mathrm{r}=0.42 ; \mathrm{p}<.001\end{array}$ & CS & $\begin{array}{l}\text { Physical activity correlates with } \\
\text { disease severity, but not with } \\
\text { fatigue or sleepiness. }\end{array}$ \\
\hline $\begin{array}{l}\text { Mills and Young } \\
2011[47]\end{array}$ & 635 & 559 valid ESS data & $46.6(10.9)$ & $71 \%$ & $7.9(4.5)$ & - & - & n.s. & CS & $\begin{array}{l}\text { Fatigue is correlated with } \\
\text { disability, disease type and } \\
\text { sleep duration (u-shaped } \\
\text { correlation). }\end{array}$ \\
\hline $\begin{array}{l}\text { Moreira } \\
\quad \text { et al., } 2008[48]\end{array}$ & 44 & $\begin{array}{l}32 \text { RLS } \\
12 \text { non-RLS }\end{array}$ & $\begin{array}{l}40.7(14.8) \\
44.1(13.4)\end{array}$ & $\begin{array}{l}67 \% \\
75 \%\end{array}$ & $\begin{array}{l}5.7(4.0) \\
5.7(3.0)\end{array}$ & $\begin{array}{l}7 \% \\
\text { total }\end{array}$ & $\begin{array}{l}- \\
-\end{array}$ & n.s. & CS & $\begin{array}{l}\text { MS patients with RLS show } \\
\text { greater disability, poorer sleep } \\
\text { and increased levels of fatigue. }\end{array}$ \\
\hline $\begin{array}{l}\text { Neau } \\
\text { et al., } 2012 \text { [49] }\end{array}$ & $\begin{array}{l}205 \\
25 \text { with PSG }\end{array}$ & $\begin{array}{l}\text { Total questionnaire } \\
8 \text { fatigue }+ \text { sleepiness } \\
17 \text { fatigue, non-sleepiness }\end{array}$ & $\begin{array}{l}43.7(11.1) \\
40.1(11.2) \\
39.7(9.3)\end{array}$ & $\begin{array}{l}76 \% \\
63 \% \\
59 \%\end{array}$ & $\begin{array}{l}7.3(4.8) \\
\mathbf{1 4 . 7}(0.5) \\
4.6(2.1)\end{array}$ & $\begin{array}{l}31 \% \\
- \\
-\end{array}$ & $\begin{array}{l}81.6(34.2)^{03} \\
118.2(23.8)^{03} \\
65.6(33.1)^{03}\end{array}$ & $\begin{array}{l}\text { FIS } \\
\mathrm{r}=0.68 \\
\text { subgroups } \mathrm{p}<.0001\end{array}$ & $\mathrm{CS}^{\mathrm{PSG}}$ & $\begin{array}{l}\text { Sleep disturbances and } \\
\text { excessive daytime sleepiness } \\
\text { are frequent in MS. }\end{array}$ \\
\hline $\begin{array}{l}\text { Neumann } \\
\text { et al., 2014 [50] }\end{array}$ & $35(15)$ & $\begin{array}{l}30 \text { fatigue } \\
5 \text { non-fatigue }\end{array}$ & $\begin{array}{l}44.7(7.1) \\
45.3(6.1)\end{array}$ & $\begin{array}{l}73 \% \\
40 \%\end{array}$ & $\begin{array}{l}8.2(2.1) \\
5.4(3.0)\end{array}$ & $\begin{array}{l}0 \%{ }^{x} \\
-\end{array}$ & $\begin{array}{l}\mathbf{7 5 . 6}(13.5)^{04} \\
\mathbf{4 9 . 4}(10.6)^{04}\end{array}$ & - & CS & $\begin{array}{l}\text { Reaction time is an objective } \\
\text { marker for fatigability. }\end{array}$ \\
\hline $\begin{array}{l}\text { Niepel } \\
\text { et al. } 2013[51]\end{array}$ & $26(9)$ & $\begin{array}{l}\text { Total } \\
17 \text { fatigue } \\
9 \text { non-fatigue }\end{array}$ & $\begin{array}{l}- \\
49.4(9.2) \\
41.8(13.1)\end{array}$ & $\begin{array}{l}65 \% \\
71 \% \\
56 \%\end{array}$ & $\begin{array}{l}5.6(4.1) \\
7.0(3.8) \\
3.0(3.7)\end{array}$ & $\begin{array}{l}15 \% \\
- \\
-\end{array}$ & $\begin{array}{l}- \\
- \\
-\end{array}$ & $\begin{array}{l}\mathrm{r}=0.41 \\
\mathrm{p}=.039 \text { in fatigued } \\
\text { patients }\end{array}$ & $\begin{array}{l}\text { CS } \\
\text { CC }\end{array}$ & $\begin{array}{l}\text { Fatigued MS patients have } \\
\text { reduced levels of alertness and } \\
\text { sympathetic activity. Modafinil } \\
\text { shows alerting and } \\
\text { sympathomimetic short-term } \\
\text { effects. }\end{array}$ \\
\hline $\begin{array}{l}\text { Papuc } \\
\text { et al., } 2010 \text { [52] }\end{array}$ & $38(15)$ & $\begin{array}{l}\text { Total } \\
10 \text { fatigue } \\
28 \text { non-fatigue }\end{array}$ & $\begin{array}{l}36^{*}[21-68] \\
34^{*}[21-69] \\
38^{*}[22-55]\end{array}$ & $\begin{array}{l}53 \% \\
54 \% \\
60 \%\end{array}$ & $\begin{array}{l}6^{*}[2-11] \\
6^{*}[2-11] \\
6^{*}[4-8]\end{array}$ & $\begin{array}{l}- \\
- \\
-\end{array}$ & $\begin{array}{l}\mathbf{5 . 5} \mathbf{5}^{*}[1.7-6.6] \\
\mathbf{5 . 7 ^ { * }}[4.8-6.6] \\
2.6^{*}[1.7-4.1]\end{array}$ & - & $\begin{array}{l}\text { CS } \\
\text { CC }\end{array}$ & $\begin{array}{l}\text { CSF hypocretin- } 1 \text { levels do not } \\
\text { differ between MS and controls, } \\
\text { but are correlated with fatigue } \\
\text { levels. }\end{array}$ \\
\hline $\begin{array}{l}\text { Pokryszko-Dragan } \\
\text { et al., 2013 [53] }\end{array}$ & 100 & $\begin{array}{l}\text { Total } \\
49 \text { fatigue } \\
51 \text { non-fatigue }\end{array}$ & $\begin{array}{l}42[20-67] \\
- \\
-\end{array}$ & $\begin{array}{l}69 \% \\
- \\
-\end{array}$ & $\begin{array}{l}6.3[0-19] \\
6.4(3.9) \\
6.2(4.7)\end{array}$ & $\begin{array}{l}19 \% \\
- \\
-\end{array}$ & $\begin{array}{l}3.8[1.1-7.0] \\
- \\
-\end{array}$ & n.s. & CS & $\begin{array}{l}\text { Sleep disturbances may } \\
\text { increase fatigue and are related } \\
\text { to MS symptoms and therapies. }\end{array}$ \\
\hline Sauter 2004 [54] & 30 & Total & $40.4(9.2)$ & $67 \%$ & $9.7(4.2)$ & $13 \%$ & $5.2(1.1)$ & n.s. & $\begin{array}{l}\text { CS } \\
\text { CC }\end{array}$ & $\begin{array}{l}\text { MS patients differ from controls } \\
\text { in sleep efficiency, sleep quality, } \\
\text { and quality of life. }\end{array}$ \\
\hline $\begin{array}{l}\text { Stanton } \\
\quad \text { et al., } 2006 \text { [55] }\end{array}$ & 60 & Total & $41^{*}[19-69]$ & $72 \%$ & $7^{*}[0-19]$ & $32 \%$ & $11^{*}[2.5-15.8]$ & $\begin{array}{l}\mathrm{FSS} \\
\mathrm{r}=0.30 ; \mathrm{p}=.022\end{array}$ & CS & $\begin{array}{l}\text { Sleep disturbances are frequent } \\
\text { in MS and may contribute to } \\
\text { fatigue. }\end{array}$ \\
\hline $\begin{array}{l}\text { Veauthier } \\
\text { et al., } 2011 \text { [56] (2013) [73] }\end{array}$ & 141 & $\begin{array}{l}66 \text { PSG total } \\
26 \text { fatigue } \\
40 \text { non-fatigue } \\
75 \text { no } \text { PSG total } \\
21 \text { fatigue } \\
54 \text { non-fatigue }\end{array}$ & $\begin{array}{l}43.2(10.0) \\
45.3(9.5) \\
42.0(10.2) \\
45.4(10.8) \\
44.5(10.6) \\
45.9(10.9)\end{array}$ & $\begin{array}{l}68 \% \\
73 \% \\
65 \% \\
67 \% \\
55 \% \\
72 \%\end{array}$ & $\begin{array}{l}8.9(4.7) \\
\mathbf{1 1 . 3}(4.2) \\
7.5(4.5) \\
8.2(4.6) \\
9.8(4.4) \\
7.6(4.5)\end{array}$ & $\begin{array}{l}- \\
- \\
- \\
- \\
- \\
-\end{array}$ & $\begin{array}{l}4.5(1.8) \\
6.0(1.0) \\
3.6(1.6) \\
4.8(1.7) \\
5.9(1.0) \\
4.4(1.7)\end{array}$ & - & $\mathrm{CS}^{\mathrm{PSG}}$ & $\begin{array}{l}\text { Significant relationship } \\
\text { between sleep disorders and } \\
\text { fatigue in MS }\end{array}$ \\
\hline $\begin{array}{l}\text { Wunderlin } \\
\text { et al., } 1997 \text { [57] }\end{array}$ & 10 & Total & $45.0(8.0)$ & $80 \%$ & $9.2(5.3)$ & $40 \%$ & $4.5(1.7)$ & - & CS & $\begin{array}{l}\text { Fatigue and daytime sleepiness } \\
\text { cannot be explained by } \\
\text { nocturnal apneas or oxygen } \\
\text { desaturations. }\end{array}$ \\
\hline
\end{tabular}




\begin{tabular}{|c|c|c|c|c|c|c|c|c|c|c|}
\hline $\begin{array}{l}\text { Authors, year } \\
\text { [reference] }\end{array}$ & $\begin{array}{l}\text { N MS-patients } \\
\text { (n controls) }\end{array}$ & $\begin{array}{l}\text { N MS-patients } \\
\text { in subgroups }\end{array}$ & $\begin{array}{l}\text { Age }(\mathrm{y}) \text { mean }(\mathrm{SD}) \\
\text { median* [range] }\end{array}$ & $\begin{array}{l}\text { Sex } \\
\text { female \% }\end{array}$ & $\begin{array}{l}\text { ESS mean (SD) } \\
\text { median* } \\
\text { [range] }\end{array}$ & ESS $>10(\%)$ & $\begin{array}{l}\text { Fatigue score } \\
\text { mean (SD) } \\
\text { median* [range] }\end{array}$ & $\begin{array}{l}\text { Significant } \\
\text { correlation } \\
\text { ESS } \times \text { fatigue score }\end{array}$ & $\begin{array}{l}\text { Study } \\
\text { design }\end{array}$ & Main outcome \\
\hline \multicolumn{11}{|l|}{ Longitudinal studies } \\
\hline $\begin{array}{l}\text { Attarian } \\
\text { et al., } 2011 \text { [58] }\end{array}$ & 29 & $\begin{array}{l}15 \text { placebo } \\
14 \text { eszopiclone }\end{array}$ & $\begin{array}{l}46.5[31-58] \\
45.0[25-64]\end{array}$ & $\begin{array}{l}80 \% \\
87 \%\end{array}$ & $\begin{array}{l}\mathbf{1 2 . 5}(4.3) \\
9.9(3.6)\end{array}$ & $\begin{array}{l}- \\
-\end{array}$ & $\begin{array}{l}11.1(2.2)^{02} \\
7.9(2.8)^{02}\end{array}$ & - & $\begin{array}{l}\text { LS } \\
\text { CC, PC }\end{array}$ & $\begin{array}{l}\text { Eszopiclone increases total } \\
\text { sleep time, but does not } \\
\text { improve fatigue in MS. }\end{array}$ \\
\hline $\begin{array}{l}\text { Brioschi } \\
\quad \text { et al., } 2009 \text { [59] }\end{array}$ & 12 & Total & $43.3(9.3)$ & $92 \%$ & $9.3(3.9)$ & - & 5.6 $(0.9)^{01}$ & - & LS & $\begin{array}{l}\text { Modafinil improves fatigue in } \\
\text { MS, no changes in physical } \\
\text { activity }\end{array}$ \\
\hline $\begin{array}{l}\text { Bruce } \\
\quad \text { et al., } 2012 \text { [60] }\end{array}$ & 30 & $\begin{array}{l}16 \text { after placebo (phase I) } \\
14 \text { after placebo (phase II) }\end{array}$ & $\begin{array}{l}49.9(7.2) \\
47.7(6.0)\end{array}$ & $\begin{array}{l}88 \% \\
79 \%\end{array}$ & $\begin{array}{l}9.3(4.3) \\
9.7(5.0)\end{array}$ & $\begin{array}{l}- \\
-\end{array}$ & $\begin{array}{l}18.6(10.2)^{05} \\
17.8(8.4)^{05}\end{array}$ & - & $\begin{array}{l}\text { LS } \\
\text { PC }\end{array}$ & $\begin{array}{l}\text { Armodafinil improves delayed } \\
\text { verbal recall, no other changes } \\
\text { in other outcome parameters. }\end{array}$ \\
\hline $\begin{array}{l}\text { Côté } \\
\text { et al., } 2013 \text { [61] }\end{array}$ & 62 & $\begin{array}{l}21 \text { SLD treated } \\
18 \text { SLD untreated } \\
17 \text { no SLD }\end{array}$ & $\begin{array}{l}51.3(8.3) \\
49.8(8.8) \\
41.9(10.7)\end{array}$ & $\begin{array}{l}62 \% \\
78 \% \\
77 \%\end{array}$ & $\begin{array}{l}9.6(3.8) \\
7.6(4.9) \\
7.9(4.8)\end{array}$ & $\begin{array}{l}- \\
- \\
-\end{array}$ & $\begin{array}{l}\mathbf{5 . 1}(1.6) \\
\mathbf{5 . 4}(1.5) \\
\mathbf{4 . 8}(1.8)\end{array}$ & - & $\mathrm{LS}^{\mathrm{PSG}}$ & $\begin{array}{l}\text { Treatment of OSA and RLS } \\
\text { improves fatigue in MS. }\end{array}$ \\
\hline $\begin{array}{l}\text { García Jalón } \\
\text { et al., } 2013 \text { [62] }\end{array}$ & 23 & $\begin{array}{l}10 \text { MS control } \\
13 \text { MS intervention }\end{array}$ & $\begin{array}{l}52.0(7.0) \\
45.9(9.9)\end{array}$ & $\begin{array}{l}60 \% \\
77 \%\end{array}$ & $\begin{array}{l}12.4(4.5) \\
6.9(4.1)\end{array}$ & $\begin{array}{l}- \\
-\end{array}$ & $\begin{array}{l}5.9(0.9) \\
5.6(0.6)\end{array}$ & - & LS & $\begin{array}{l}\text { High acceptance of an energy } \\
\text { conservation program by MS } \\
\text { patients }\end{array}$ \\
\hline Gerhard 2009 [63] & 30 & Total & $36.0(10.4)$ & $67 \%$ & $8.4(3.7)$ & $37 \%$ & $35.2(16.9)^{1}$ & - & LS & $\begin{array}{l}\text { IFN } \beta 1 \text { a treatment increases } \\
\text { fatigue and improves cognitive } \\
\text { functions. }\end{array}$ \\
\hline $\begin{array}{l}\text { Kallweit } \\
\text { et al., } 2013 \text { [64] }\end{array}$ & 69 & $\begin{array}{l}\text { Total } \\
28 \text { SRBD } \\
41 \text { non-SRBD }\end{array}$ & $\begin{array}{l}49.8(9.2) \\
53.3(9.5) \\
47.4(8.3)\end{array}$ & $\begin{array}{l}70 \% \\
57 \% \\
78 \%\end{array}$ & $\begin{array}{l}- \\
9.7(3.8) \\
9.4(4.7)\end{array}$ & $\begin{array}{l}- \\
61 \% \\
44 \%\end{array}$ & $\begin{array}{l}- \\
\mathbf{5 . 5}(0.9) \\
\mathbf{5 . 7}(0.7)\end{array}$ & - & LS & $\begin{array}{l}\text { High prevalence of SRBD in MS } \\
\text { patients; continuous positive } \\
\text { airway pressure therapy } \\
\text { decreases fatigue but not } \\
\text { sleepiness. }\end{array}$ \\
\hline $\begin{array}{l}\text { Mendozzi } \\
\text { et al., } 2010 \text { [65] }\end{array}$ & 42 & $\begin{array}{l}\text { Total } \\
12 \text { no-IMA } \\
10 \text { glatiramer acetate } \\
10 \text { IFN } \beta 1 \text { a/b s.c. } \\
10 \text { IFN } \beta 1 \text { a/b i.m.. }\end{array}$ & $\begin{array}{l}39.4(7.4) \\
41.8(5.7) \\
38.6(8.4) \\
38.5(9.9) \\
38.2(5.4)\end{array}$ & $\begin{array}{l}- \\
- \\
- \\
- \\
-\end{array}$ & $\begin{array}{l}5.7(3.2) \\
6.3(3.6) \\
6.8(2.5) \\
4.8(2.7) \\
4.9(3.6)\end{array}$ & $\begin{array}{l}14 \% \\
- \\
- \\
- \\
-\end{array}$ & $\begin{array}{l}3.6(1.8) \\
3.4(1.6) \\
\mathbf{4 . 4}(1.7) \\
\mathbf{4 . 1}(1.9) \\
2.7(1.6)\end{array}$ & - & LS & $\begin{array}{l}\text { IFN } \beta \text { and glatiramer acetate } \\
\text { treatment decrease sleep } \\
\text { efficiency in MS assessed by } \\
\text { actigraphy }\end{array}$ \\
\hline $\begin{array}{l}\text { Möller } \\
\text { et al., } 2011 \text { [66] }\end{array}$ & 121 & $\begin{array}{l}59 \text { placebo } \\
62 \text { modafinil }\end{array}$ & $\begin{array}{l}40.8(11.2) \\
41.4(9.5)\end{array}$ & $\begin{array}{l}78 \% \\
63 \%\end{array}$ & $\begin{array}{l}11.8(5.0) \\
11.8(4.9)\end{array}$ & $\begin{array}{l}- \\
-\end{array}$ & $\begin{array}{l}5.8(0.8) \\
6.0(0.8)\end{array}$ & - & $\begin{array}{l}\text { LS } \\
\text { PC }\end{array}$ & $\begin{array}{l}\text { No effect of modafinil on fatigue } \\
\text { in MS }\end{array}$ \\
\hline $\begin{array}{l}\text { Rammohan } \\
\text { et al., } 2002 \text { [67] }\end{array}$ & 72 & Total & $44.0[23-61]$ & $75 \%$ & $9.5[1-20]$ & - & $5.9[4-7]$ & - & $\begin{array}{l}\text { LS } \\
\text { PC }\end{array}$ & $\begin{array}{l}\text { Modafinil significantly } \\
\text { improves fatigue. }\end{array}$ \\
\hline $\begin{array}{l}\text { Stankoff } \\
\quad \text { et al., } 2005 \text { [68] }\end{array}$ & 115 & $\begin{array}{l}59 \text { placebo } \\
56 \text { modafinil }\end{array}$ & $\begin{array}{l}44.0(9.0) \\
43.8(8.0)\end{array}$ & $\begin{array}{l}- \\
-\end{array}$ & $\begin{array}{l}9.7(5.5) \\
\mathbf{1 0 . 6}(4.8)\end{array}$ & $\begin{array}{l}53 \% \\
\text { total }\end{array}$ & $\begin{array}{l}\mathbf{6 3 . 1}(9.3)^{05} \\
\mathbf{6 3 . 3}(10.0)^{05}\end{array}$ & - & $\begin{array}{l}\text { LS } \\
\text { PC }\end{array}$ & $\begin{array}{l}\text { No differences between effects } \\
\text { of modafinil and placebo } \\
\text { treatment on fatigue in MS }\end{array}$ \\
\hline $\begin{array}{l}\text { Svenningsson } \\
\text { et al., 2013 [69] }\end{array}$ & 195 & Total & $39.7(9.2)$ & $71 \%$ & $8.8(-)$ & - & $71.2(-)^{04}$ & - & LS & $\begin{array}{l}\text { Natalizumab improves fatigue, } \\
\text { sleepiness, quality of life, } \\
\text { depression, and cognition. }\end{array}$ \\
\hline $\begin{array}{l}\text { Vakhapova } \\
\text { et al., } 2010 \text { [70] }\end{array}$ & 16 & Total & $45.4[26-60]$ & - & $\begin{array}{l}6.4(5.6) \\
\text { after placebo }\end{array}$ & - & $\begin{array}{l}37.4(15.8)^{1} \\
\text { after placebo }\end{array}$ & - & $\begin{array}{l}\text { LS } \\
\text { PC }\end{array}$ & $\begin{array}{l}\text { Sublingual tizanidine improves } \\
\text { daytime sleepiness and } \\
\text { spasticity in MS }\end{array}$ \\
\hline $\begin{array}{l}\text { Van Kessel } \\
\quad \text { et al. }{ }^{+} 2008 \text { [71] }\end{array}$ & 72 & $\begin{array}{l}35 \mathrm{CBT} \\
37 \text { relaxation training }\end{array}$ & $\begin{array}{l}42.9(9.3) \\
47.0(9.5)\end{array}$ & $\begin{array}{l}80 \% \\
70 \%\end{array}$ & $\begin{array}{l}6^{*}[-] \\
5^{*}[-]\end{array}$ & $\begin{array}{l}- \\
-\end{array}$ & $\begin{array}{l}20.9^{1}(4.3) \\
20.3^{1}(4.3)\end{array}$ & - & $\begin{array}{l}\text { LS } \\
\text { CC }\end{array}$ & $\begin{array}{l}\text { Both CBT and relaxation } \\
\text { training are effective } \\
\text { treatments for fatigue in MS; } \\
\text { CBT is more effective. }\end{array}$ \\
\hline $\begin{array}{l}\text { Zifko } \\
\quad \text { et al., } 2002 \text { [72] }\end{array}$ & 50 & Total & $40.4(10.3)$ & $60 \%$ & $9.7(3.9)$ & - & $30.3(8.5)^{1}$ & - & LS & $\begin{array}{l}\text { Modafinil treatment improves } \\
\text { fatigue and sleepiness in MS. }\end{array}$ \\
\hline
\end{tabular}

Symbols: *median; ${ }^{* *}$ median [interquartile range]; longitudinal study; ${ }^{+}$data provided on request; ${ }^{\text {PSG }}$ use of PSG; ${ }^{\text { }}$ ESS score $>10$ exclusion criterion; - data not reported.

Statistics: F/non-F: subdivision in fatigued and non-fatigued subgroups; n: number of participants; n.s.: not significant; p: p-value; r: correlation coefficient; SD: standard deviation.

Fatigue Scales: FSS: fatigue severity scale (cut off $>4$ ); ${ }^{1}$ FSS total score (cut off $>36$ ).

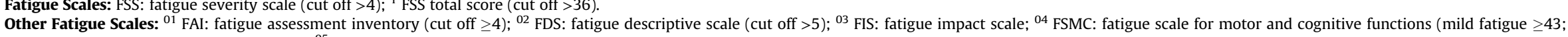
moderate fatigue $\geq 53$; severe fatigue $\geq 63$ ); ${ }^{05}$ MFIS: modified fatigue impact scale (cut off $>34$ ).

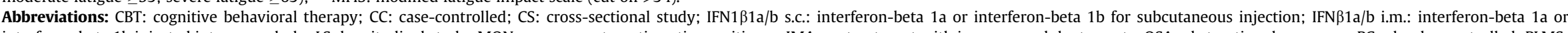

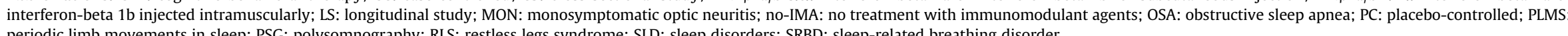
periodic limb movements in sleep; PSG: polysomnography; RLS: restless legs syndrome; SLD: sleep disorders; SRBD: sleep-related breathing disorder.

Boldfaced numerals denote scores above critical cut offs (e.g., ESS $>10$; FSS $>4$ ); Italic printed numerals mark fatigue scores other than FSS score. 
Additional information on longitudinal studies using the Epworth sleepiness scale (ESS) as an assessment tool in patients with multiple sclerosis (MS).

\begin{tabular}{|c|c|c|c|c|c|c|c|c|}
\hline $\begin{array}{l}\text { Authors, year } \\
\text { [reference] }\end{array}$ & N MS-patients & $\begin{array}{l}\text { ESS baseline mean } \\
\text { (SD) median* } \\
\text { [range] }\end{array}$ & $\begin{array}{l}\text { ESS follow-up/ } \\
\text { intervention } \\
\text { mean (SD)/median* } \\
\text { [range] }\end{array}$ & $\begin{array}{l}\text { Fatigue baseline } \\
\text { mean (SD) }\end{array}$ & $\begin{array}{l}\text { Fatigue follow-up/ } \\
\text { intervention } \\
\text { mean (SD) }\end{array}$ & $\begin{array}{l}\text { Follow-up } \\
\text { period to baseline }\end{array}$ & Intervention & Intervention efficacy \\
\hline $\begin{array}{l}\text { Attarian } \\
\quad \text { et al., } 2011 \text { [58] }\end{array}$ & $\begin{array}{l}15 \text { placebo } \\
14 \text { eszopiclone }\end{array}$ & $\begin{array}{l}\mathbf{1 2 . 5}(4.3) \\
9.9(3.6)\end{array}$ & $\begin{array}{l}\mathbf{1 0 . 5}(3.9) \\
8.2(4.0)\end{array}$ & $\begin{array}{l}\mathbf{1 1 . 1}^{02}(2.2) \\
7.9^{02}(2.8)\end{array}$ & $\begin{array}{l}5.4^{02}(3.1) \\
4.6^{02}(3.6)\end{array}$ & $7 \mathrm{wk}$ & Eszopiclone or placebo & $\begin{array}{l}\text { n.s. ESS and FSS } \\
\text { changes }\end{array}$ \\
\hline $\begin{array}{l}\text { Brioschi } \\
\quad \text { et al., } 2009 \text { [59] }\end{array}$ & 12 & $9.3(-)$ & $\begin{array}{l}\text { T1: } 9.1(3.0) \\
\text { T2: 10.4 }(4.7)\end{array}$ & $5.6^{01}(0.9)$ & $\begin{array}{l}\text { T1: } 4.5^{01}(1.1) \\
\text { T2: } 5.1^{01}(1.1)\end{array}$ & $\begin{array}{l}\text { T1: } 3 \text { mo with modafinil } \\
\text { T2: } 1 \text { mo without modafinil }\end{array}$ & Modafinil & $\begin{array}{l}\text { n.s. ESS changes; } \\
\text { significant FAI } \\
\text { improvements T0 to T1 } \\
(\Delta 1.1)\end{array}$ \\
\hline $\begin{array}{l}\text { Bruce } \\
\quad \text { et al., } 2012 \text { [60] }\end{array}$ & $\begin{array}{l}16 \text { placebo first } \\
14 \text { armodafinil first }\end{array}$ & $\begin{array}{l}9.3(4.3) \\
9.7(5.0)\end{array}$ & $\begin{array}{l}9.2(5.0) \\
\mathbf{1 0 . 5}(4.3)\end{array}$ & $\begin{array}{l}18.6^{05}(10.2) \\
17.8^{05}(8.4)\end{array}$ & $\begin{array}{l}18.2^{05}(10.6) \\
18.6^{05}(10.5)\end{array}$ & $\begin{array}{l}1 \text { wk change } \\
\text { (cross-over design) }\end{array}$ & Armodafinil & $\begin{array}{l}\text { n.s. ESS and FSS } \\
\text { changes }\end{array}$ \\
\hline $\begin{array}{l}\text { Côté } \\
\text { et al., } 2013 \text { [61] }\end{array}$ & $\begin{array}{l}21 \text { SLD, treated } \\
18 \text { SLD, untreated } \\
17 \text { no SLD }\end{array}$ & $\begin{array}{l}9.6(3.8) \\
7.6(4.9) \\
7.9(4.8)\end{array}$ & $\begin{array}{l}6.1(3.8) \\
7.1(5.5) \\
7.2(4.2)\end{array}$ & $\begin{array}{l}\mathbf{5 . 1}(1.6) \\
\mathbf{5 . 4}(1.5) \\
\mathbf{4 . 8}(1.8)\end{array}$ & $\begin{array}{l}\mathbf{4 . 5}(1.7) \\
\mathbf{5 . 1}(1.5) \\
\mathbf{5 . 2}(1.2)\end{array}$ & $\geq 3 \mathrm{mo}$ & $\begin{array}{l}\text { Treatment of OSA and } \\
\text { RLS }\end{array}$ & $\begin{array}{l}\text { Significant FSS }(\Delta 0.9) \text {, } \\
\text { MFI }{ }^{06} \text {, ESS and PSQI } \\
\text { improvements }\end{array}$ \\
\hline $\begin{array}{l}\text { García Jalón } \\
\quad \text { et al., } 2013 \text { [62] }\end{array}$ & 13 MS intervention & $12.4(4.5)$ & $\begin{array}{l}\text { T1: } \mathbf{1 0 . 2}(4.3) \\
\text { T2: } \mathbf{1 4 . 0}(10.7) \\
\text { T3: } \mathbf{1 0 . 6}(4.2) \\
\text { T1: } 6.6(4.2) \\
\text { T2: } 6.5(4.9) \\
\text { T3: } 6.9(4.1)\end{array}$ & $\mathbf{5 . 9}(0.9)$ & $\begin{array}{l}\text { T1: } 4.9(1.0) \\
\text { T2: } \mathbf{5 . 5}(0.9) \\
\text { T3: } \mathbf{4 . 9}(1.3) \\
\text { T1: } \mathbf{5 . 0}(1.4) \\
\text { T2: } \mathbf{4 . 7}(1.7) \\
\text { T3: } \mathbf{5 . 2}(1.3)\end{array}$ & $\begin{array}{l}\text { T1: intervention } \\
\text { T2: } 6 \mathrm{wk} \\
\text { T3: } 3 \mathrm{mo}\end{array}$ & $\begin{array}{l}\text { Energy conservation } \\
\text { program }\end{array}$ & $\begin{array}{l}\text { n.s. ESS, FSS and FIS } \\
\text { changes; significant FIS } \\
\text { Cognitive } \\
\text { improvements }(\Delta 5.8)\end{array}$ \\
\hline $\begin{array}{l}\text { Gerhard } \\
\quad 2009[63]\end{array}$ & 30 & $8.4(3.7)$ & $10.2(4.4)$ & $35.2^{1}(16.9)$ & $38.8^{1}(16.8)$ & $\geq 6 \mathrm{mo}$ & Interferon & $\begin{array}{l}\text { Significant ESS }(\Delta 1.6) \\
\text { and FSS }(\Delta 3.6) \text { decline }\end{array}$ \\
\hline $\begin{array}{l}\text { Kallweit } \\
\quad \text { et al., } 2013 \text { [64] }\end{array}$ & $\begin{array}{l}28 \text { SRBD } \\
41 \text { non-SRBD }\end{array}$ & $\begin{array}{l}9.7(3.8) \\
9.4(4.7)\end{array}$ & $\begin{array}{l}9.5(3.0) \\
-\end{array}$ & $5.5(0.9) 5.7(0.7)$ & $\begin{array}{l}4.8(0.6) \\
-\end{array}$ & $\geq 6 \mathrm{mo}$ & OSA treatment & $\begin{array}{l}\text { n.s. ESS changes; } \\
\text { significant FSS } \\
\text { improvements }(\Delta 1.0)\end{array}$ \\
\hline $\begin{array}{l}\text { Mendozzi } \\
\text { et al., } 2010 \text { [65] }\end{array}$ & $\begin{array}{l}42 \text { total } \\
12 \text { no-IMA } \\
10 \text { GA } \\
10 \text { IFN } \beta 1 \text { a/b s.c. } \\
10 \text { IFN } \beta 1 \text { a/b i.m. }\end{array}$ & $\begin{array}{l}5.7(3.2) \\
6.3(3.6) \\
6.8(2.5) \\
4.8(2.7) \\
4.9(3.6)\end{array}$ & $\begin{array}{l}5.6(3.9) \\
6.4(3.9) \\
7.6(3.6) \\
4.8(4.4) \\
3.5(2.5)\end{array}$ & $\begin{array}{l}3.6(1.8) \\
3.4(1.6) \\
\mathbf{4 . 4}(1.7) \\
\mathbf{4 . 1}(1.9) \\
2.7(1.6)\end{array}$ & $\begin{array}{l}3.7(1.7) \\
3.5(1.3) \\
\mathbf{4 . 5}(1.9) \\
4.0(1.6) \\
2.8(1.6)\end{array}$ & $\geq$ seven nights & $\begin{array}{l}\text { No-IMA, GA, IFN } \beta 1 \mathrm{a} / \mathrm{b} \\
\text { s.c., IFN } \beta 1 \mathrm{a} / \mathrm{b} \text { i.m. }\end{array}$ & $\begin{array}{l}\text { n.s. ESS and FSS } \\
\text { changes }\end{array}$ \\
\hline $\begin{array}{l}\text { Möller } \\
\text { et al., } 2011 \text { [66] }\end{array}$ & $\begin{array}{l}62 \text { modafinil } \\
59 \text { placebo }\end{array}$ & $\begin{array}{l}11.8(4.9) \\
11.8(5.0)\end{array}$ & $\begin{array}{l}9.7(4.4) \\
9.5(4.9)\end{array}$ & $\begin{array}{l}\text { 6.0 }(0.8) \\
\mathbf{5 . 8}(0.8)\end{array}$ & $\begin{array}{l}\mathbf{5 . 3}(1.2) \\
\mathbf{5 . 4}(1.0)\end{array}$ & $8 \mathrm{wk}$ & Modafinil & $\begin{array}{l}\text { n.s. ESS, FSS and MFIS } \\
\text { changes }\end{array}$ \\
\hline $\begin{array}{l}\text { Rammohan } \\
\text { et al., } 2002 \text { [67] }\end{array}$ & 72 & $9.5[1-20]$ & $\begin{array}{l}\text { T1: } 7.2(-) \\
\text { T2: } 7.0(-) \\
\text { T3: }-\end{array}$ & $5.9[4-7]$ & $\begin{array}{l}\text { T1: } 4.7(-) \\
\text { T2: } 5.3(-) \\
\text { T3: } 5.3(-)\end{array}$ & $\begin{array}{l}\text { T1: } 2 \text { wk } 200 \mathrm{mg} \text { modafinil } \\
\text { T2: } 4 \mathrm{wk}(2 \mathrm{wk} 400 \mathrm{mg}) \\
\text { T3: } 7 \mathrm{wk}(3 \mathrm{wk} \text { placebo } \\
\text { washout) }\end{array}$ & Modafinil & $\begin{array}{l}\text { T1: significant ESS } \\
(\Delta 2.3) \text { and FSS }(\Delta 0.8) \\
\text { improvements; } \\
\text { T2: significant ESS } \\
\text { improvements }(\Delta 2.5) \text {; } \\
\text { n.s. FSS changes }\end{array}$ \\
\hline $\begin{array}{l}\text { Stankoff } \\
\quad \text { et al., } 2005 \text { [68] }\end{array}$ & $\begin{array}{l}56 \text { modafinil } \\
59 \text { placebo }\end{array}$ & $\begin{array}{l}\mathbf{1 0 . 6}(4.8) \\
9.7(5.5)\end{array}$ & - & $\begin{array}{l}63.3^{03}(10.0) \\
63.1^{03}(9.3)\end{array}$ & $\begin{array}{l}52.3^{05}(18.5) \\
49.2^{05}(16.6)\end{array}$ & $35 \mathrm{~d}$ & Modafinil & $\begin{array}{l}\text { Significant MFIS } \\
\text { improvements for both } \\
\text { groups (modafinil: } \\
\Delta 11.0 \text { vs. placebo: } \\
\Delta 13.9 \text { ), but no benefit of } \\
\text { modafinil compared to } \\
\text { placebo }\end{array}$ \\
\hline $\begin{array}{l}\text { Svenningsson } \\
\text { et al., 2013 [69] }\end{array}$ & 143 & $8.8(-)$ & $7.5(-)$ & $71.2^{04}(-)$ & $62.2^{04}(-)$ & $12 \mathrm{mo}$ & Natalizumab & $\begin{array}{l}\text { Significant ESS }(\Delta 1.33) \\
\text { and FSMC }(\Delta 9.0) \\
\text { improvements }\end{array}$ \\
\hline $\begin{array}{l}\text { Vakhapova } \\
\text { et al., } 2010 \text { [70] }\end{array}$ & 16 & $\begin{array}{l}6.4(5.6) \\
\text { after placebo phase }\end{array}$ & $\begin{array}{l}4.8(4.6) \text { s.l. } \\
5.5(4.6) \text { oral }\end{array}$ & $\begin{array}{l}\mathbf{3 7 . 4}^{\mathbf{1}}(15.8) \\
\text { after placebo phase }\end{array}$ & $\begin{array}{l}33.6^{1}(16.8) \text { s.l. } \\
34.1^{1}(17.0) \text { oral }\end{array}$ & $\begin{array}{l}7 \mathrm{~d} \text { placebo phase and } 7 \mathrm{~d} \\
\text { of each condition } \\
\text { (cross-over design) }\end{array}$ & Tizanidine & $\begin{array}{l}\text { Significant ESS } \\
\text { improvements }(\Delta 1.6 \\
\text { only for s.l. application } \\
\text { vs. placebo) }\end{array}$ \\
\hline
\end{tabular}




\begin{tabular}{|c|c|c|c|c|c|c|c|c|}
\hline $\begin{array}{l}\text { Authors, year } \\
\text { [reference] }\end{array}$ & N MS-patients & $\begin{array}{l}\text { ESS baseline mean } \\
\text { (SD) median* } \\
\text { [range] }\end{array}$ & $\begin{array}{l}\text { ESS follow-up/ } \\
\text { intervention } \\
\text { mean (SD)/median* } \\
\text { [range] }\end{array}$ & $\begin{array}{l}\text { Fatigue baseline } \\
\text { mean }(\mathrm{SD})\end{array}$ & $\begin{array}{l}\text { Fatigue follow-up/ } \\
\text { intervention } \\
\text { mean (SD) }\end{array}$ & $\begin{array}{l}\text { Follow-up } \\
\text { period to baseline }\end{array}$ & Intervention & Intervention efficacy \\
\hline $\begin{array}{l}\text { Van Kessel } \\
\quad \text { et al. }^{+} 2008 \text { [71] }\end{array}$ & $\begin{array}{l}35 \mathrm{CBT} \\
37 \text { relaxation training }\end{array}$ & $\begin{array}{l}6^{*}[-] \\
5^{*}[-]\end{array}$ & $\begin{array}{l}\text { T1: } 3^{*}[-] \\
\text { T2: } 3^{*}[-] \\
\text { T3: } 3^{*}[-] \\
\text { T1: } 6^{*}[-] \\
\text { T2: } 4^{*}[-] \\
\text { T3: } 4^{*}[-]\end{array}$ & $\begin{array}{l}20.9^{1}(4.3) \\
20.3^{1}(4.3)\end{array}$ & $\begin{array}{l}\text { T1: } 7.9^{1}(4.3) \\
\text { T2: } 9.0^{1}(5.3) \\
\text { T3: } 10.4^{1}(6.4) \\
\text { T1: } 11.6^{1}(5.3) \\
\text { T2:11. } 1^{1}(4.6) \\
\text { T3:12.5 }(5.2)\end{array}$ & $\begin{array}{l}\text { T1: } 2 \mathrm{mo} \text { (post treatment) } \\
\text { T2: } 5 \mathrm{mo} \\
\text { T3: } 8 \mathrm{mo}\end{array}$ & $\begin{array}{l}\text { CBT vs. relaxation } \\
\text { training }\end{array}$ & $\begin{array}{l}\text { Significantly greater } \\
\text { fatigue reductions in } \\
\text { the CBT group across } \\
\text { the } 8 \text { mo compared to } \\
\text { the relaxation training } \\
\text { group }\end{array}$ \\
\hline $\begin{array}{l}\text { Veauthier } \\
\text { et al., } 2013 \text { [73] }\end{array}$ & $\begin{array}{l}58 \text { total } \\
13 \text { good compliance } \\
12 \text { moderate } \\
\text { compliance } \\
17 \text { no compliance } \\
4 \text { no feedback } \\
12 \text { no sleep disorder }\end{array}$ & $\begin{array}{l}9.1(4.6) \\
9.5(5.9) \\
9.8(4.8) \\
\\
\mathbf{1 0 . 8}(3.7) \\
6.0(4.5) \\
6.4(2.9)\end{array}$ & $\begin{array}{l}8.5(4.6) \\
9.4(5.9) \\
9.3(5.9) \\
\\
\mathbf{1 0 . 2}(2.9) \\
6.8(3.3) \\
5.7(2.5)\end{array}$ & $\begin{array}{l}\mathbf{4 . 7}(2.3) \\
\mathbf{4 . 8}(1.1) \\
5.5(3.9) \\
\mathbf{4 . 8}(1.7) \\
\mathbf{4 . 9}(1.5) \\
3.3(1.5)\end{array}$ & $\begin{array}{l}\mathbf{4 . 0}(1.8) \\
\mathbf{4 . 3}(1.5) \\
3.9(2.2) \\
\mathbf{4 . 4}(1.7) \\
3.2(1.9) \\
3.4(1.6)\end{array}$ & 16 mo (median) & $\begin{array}{l}\text { Treatment of sleep } \\
\text { disorders }\end{array}$ & $\begin{array}{l}\text { n.s. ESS changes; } \\
\text { significant FSS } \\
\text { improvements on in } \\
\text { the entire cohort }(\Delta 0.7) \text {, } \\
\text { but n.s. changes in } \\
\text { subgroups; significant } \\
\text { MFIS improvements in } \\
\text { good compliance } \\
\text { subgroup }(\Delta 15)\end{array}$ \\
\hline Zifko et al., 2002 [72] & 50 & $9.7(3.9)$ & $4.9(2.9)$ & $30.3^{1}(8.5)$ & $25.4^{1}(3.7)$ & $3 \mathrm{mo}$ & Modafinil & $\begin{array}{l}\text { Significant ESS }(\Delta 4.8) \\
\text { and FSS }(\Delta 4.9) \\
\text { improvement }\end{array}$ \\
\hline
\end{tabular}

Statistics: n: number of participants; n.s.: not significant; SD: standard deviation; T1-3: times of assessment; $\Delta$ : changes; - data not reported.

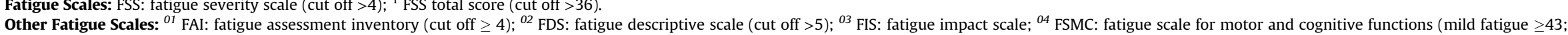
moderate fatigue $\geq 53$; severe fatigue $\geq 63$ ); ${ }^{05}$ MFIS: modified fatigue impact scale (cut off $>34 / 45$ ); ${ }^{06}$ MFI: multidimensional fatigue inventory.

Alo An revitis: intramuscularly; no-lMA: no treatment with immunomodulant agents; OSA: Obstructive sleep apnea; PSQI: Pittsburgh sleep quality index; RLS: restless legs syndrome; SRBD: sleep-related breathing disorder; SLD: sleep

Boldfaced numerals denote scores above critical cut offs (e.g., ESS >10; FSS >4); Italic printed numerals mark fatigue scores measured with fatigue scales other than FSS. 
supplement. The reference lists of articles that were regarded eligible were scanned by AF, NK, and RP.

\section{Eligibility assessment}

Eligibility assessment was performed independently in a standardized manner by three reviewers (RP, AF, and NK). Disagreements were solved by consensus. If no agreement could be reached, another author (TW) was designated to make the final decision. Studies were included only if they contained the following: a) original investigations - articles in which no original data was published were excluded (e.g., mostly reviews, letters to the editor etc.) b) published ESS data - the article had to provide statistical data of the ESS score (e.g., mean, median) c) adult ( $\geq 18 \mathrm{y}$ ) patients with MS d) sufficient sample sizes, $(n \geq 10)$ that allowed for inferential statistics, i.e., no case reports or studies providing only descriptive data. Finally, no restrictions concerning duration of illness, subtype of MS or other specifications were applied. We also did not limit this review to studies with special interventions.

\section{Data extraction}

A data-extraction sheet was developed and pilot-tested on 10 pseudo-randomly selected articles. After applying some adaptive changes, it was used for extracting information from all included studies (see Table S1 - Appendix 3). The data extraction was performed by one of the authors (AF) and examined by another (NK). If there were any ambiguities concerning the data, they was discussed by a committee of three additional authors (RP, RW, and TCW). From each study we extracted the information presented in Tables 2 and 3.

\section{Results}

\section{Study selection}

Disagreements regarding eligibility assessment between the reviewers (AF, RP, and NK) were minor and could be resolved by consensus. Our cross-database search yielded 607 records. After removing duplicates, 521 records remained and were screened for relevance. 440 records were excluded because no original data were published, disorders other than MS were investigated (e.g., sleep disorders, Parkinson's disease), or there were no ESS data specified in the abstract and no full text articles were available. If the record was categorized as potentially relevant, the full text article was assessed for eligibility. 21 studies were not included because the ESS was not used as an assessment tool. In three studies, the number of participants was too small $(<10)$ to be included. One of the full text articles was excluded because the patients were younger than 18 $y$ of age. Ten of the full text articles were excluded because no sufficient statistical ESS data were published. However, if the corresponding author of these studies could provide ESS data on request, the articles were included in the study selection $(n=5$; see supplementary material - Appendix 2). Finally, our systematic review comprised 48 studies (Table 2) [26-73].

The flow diagram of the selection process is presented in Fig. 1. Detailed information on articles excluded by full-text review is given in Appendix 4 of the supplementary material.

\section{Review findings}

No systematic epidemiological studies on the prevalence of daytime sleepiness in MS patients using the ESS as a main outcome parameter have been published so far. Out of 48 original articles, about one-third (30\%) of the studies comprised a larger MS sample size, i.e., at least 100 patients were included. The majority of articles
(67\%) had a cross-sectional study design, while the others, in most cases, used a long-term longitudinal study design to assess different treatment effects of medication and other interventions such as treatment of sleep disorders [58-72]. In particular, two studies investigated the effect of continuous positive airway pressure (CPAP) treatment on respiratory disturbances occurring during sleep, which are defined as sleep-related breathing disorder (SRBD) $[61,64]$. As the ESS and FSS are trait-specific questionnaires, potential improvements of both scores depend on lasting interventions (i.e., from one week to several months). Thus, we did not consider temporary pharmacological effects lasting only a few hours as longitudinal data [51]. We also used baseline results of longitudinal studies to gain cross-sectional data on ESS and fatigue scores. Out of all investigations, 19 studies provided data on the correlation between the ESS and the FSS (89\%) or other fatigue scales (e.g., NFI-MS, MFIS). Finally, for objective measures of sleep disorders in MS patients, nine studies using polysomnography (PSG) $[27,30,33,36,40,41,49,56,61]$ and two studies applying actigraphy $[26,65]$ were used.

\section{Cross-sectional data}

Among the studies reporting on fatigue levels ( $n=40$ ), increased fatigue scores (e.g., FSS $>4$ or MFIS $>34$ ) were found in the vast majority (78\%) and were mostly well above their critical cut-offs. Increased daytime sleepiness, as defined by a mean ESS score above 10, was detected in 11 studies (23\%) reporting ESS values in either total samples or subgroups of patients. The largest non-systematic survey of sleep disorders in the MS population to date, comprising 2375 patients, suggests increased daytime sleepiness in $30 \%$ of the respondents based on an ESS score above 10 [31] (see Table 2). Within the patient groups, elevated mean ESS scores mostly ranged from 10.5 to 12.5 and were close to their critical cutoff value. Only a single study, with 21 fatigued MS patients, showed a higher mean ESS score for the whole group (15.7) [33]. On an individual level, the proportion of MS patients with elevated ESS scores was up to $61 \%$ in those studies that provided frequency data (23 studies in total). Six studies with population sizes of at least 100 MS-patients reported frequencies of increased ESS scores between $19 \%$ and $53 \%$.

Among the 19 studies that investigated the correlation between ESS and fatigue scores, six found no significant correlation between the two $[28,41,47,48,53,54]$. The other studies reported low [45] to high correlations [33] (from $r=0.18$ to 0.74 ) with most correlations being in the moderate range $(\mathrm{r}=0.30$ to 0.47 ) $[30,34,35,38,40,44,46,51,55]$. Several studies using PSG or actigraphy found an association between an increased level of sleepiness or fatigue and objectively measured sleep disorders such as disrupted sleep/abnormal sleep cycles [26], impaired sleep quality $[33,41,49,56]$, periodic limb movement disorder (PLMD) [27] or SRBD $[40,61]$. Only one polysomnographic study did not find a difference in the sleep parameters between the fatigued versus non-fatigued MS-patients [36].

\section{Longitudinal data}

Our search yielded 16 studies reporting ESS data at baseline and at follow-up (see Table 3). Six studies specifically evaluated the efficacy of wakefulness-promoting agents (e.g., modafinil or armodafinil) on fatigue or sleepiness. In two placebo-controlled studies, modafinil [66] or armodafinil [60] did not significantly improve fatigue or sleepiness at all. Among two further studies using placebo, one demonstrated a substantial placebo effect on fatigue with no additional benefit from modafinil [68], whereas the other study only reported significant improvements in fatigue compared to the placebo run-in phase [67]. Two non-placebo-controlled studies with modafinil showed significant improvement on either both fatigue 
and sleepiness [72] or fatigue alone [59]. The impact of other medications focusing on various clinical effects (e.g., on sleep quality or as disease-modifying immunotherapies) was assessed by five other studies. In two placebo-controlled studies, the hypnotic agent eszopiclone had no significant effect on ESS or FSS [58], while the nightly sublingual administration of the antispasmodic drug tizanidine significantly reduced next-day spasticity and was associated with a reduction of the ESS-score. This effect was unexpected, since day-dose tizanidine typically increases daytime hypersomnolence [70]. In three observational studies involving immune modulatory drugs, natalizumab improved both sleepiness and fatigue [69], whereas interferon-beta, which may provoke sleep disturbances, did not change [65] or even negatively affected both conditions in the long term [63]. The injection of interferon-beta impaired sleep efficiency during the following night and led to short-term effects of sleepiness and fatigue during the next day [65].

Three of the 16 longitudinal studies investigated the effects of sleep disorder treatment. One study showed a significant improvement of both fatigue and sleepiness, particularly for the treatment of SRBD [61]. Two other studies found that either CPAPtherapy [64] or treatment of different sleep disorders (e.g. RLS, PLMD, insomnia, SRBD) [73] were associated with a significant but moderate decrease of FSS scores, whereas ESS scores did not change. Participants of a cognitive behavioral therapy group improved significantly more over time (6 mo follow-up) than participants of relaxation training, in terms of fatigue as assessed by the FSS. Such an improvement was not found for the ESS [71]. In a single study, an energy conservation program to manage fatigue was tested [62]. Significant improvement of cognitive scores on the fatigue impact scale (FIS) could be observed, but no significant changes in all other outcome variables, including FSS and ESS, were found.

\section{Discussion}

In our comprehensive literature search we strived to find all available evidence on the use of the ESS as an assessment tool in adult patients with MS. Finally, we identified 48 studies that fulfilled our inclusion criteria.

The majority of studies showed clinically significant fatigue in MS, whereas sleepiness was reported to be less frequent and less severe. Nevertheless, a substantial number of fatigued patients showed increased ESS scores as well. Some studies investigating the association between sleepiness and fatigue revealed weak to moderate correlations between both conditions, mainly depending on the presence of comorbid sleep disorders. In general, sleep disorders such as SRBD [61,74] or PLMD [27] and impaired sleep quality are often associated with fatigue or sleepiness [33,56]. Importantly, the small number of available placebo-controlled, interventional studies showed that wakefulness-promoting agents, such as modafinil or armodafinil, were not consistently effective to reduce either sleepiness or fatigue in the long-term $[60,66,68]$. These findings are in line with a 2015 Cochrane database review reporting weak and inconclusive evidence for the efficacy of stimulants, such as modafinil, pemoline or amantadine, as pharmacological treatments for fatigue in MS [75].

\section{The extent of sleepiness and fatigue in MS}

In almost all (85\%) selected studies using the ESS as an assessment tool, fatigue scores (typically measured with the FSS) were also presented. Increased fatigue in patients with MS, as indicated by elevated fatigue rating scores, was present in the majority (78\%) of the reviewed studies that provided fatigue data. This finding is in accordance with previous epidemiological studies that showed high prevalence of fatigue in patients with MS [7-10].
In contrast, increased daytime sleepiness (mean ESS score $>10$ ) was observed less frequently (26\% of the studies), comprising a limited number of patients with MS (see Table 2). Two studies reporting mean ESS scores of 15.0 and 15.7 referred to two subgroups that were $a$ priori defined as sleepy or fatigued comprising only 6 (total $n=12$ ) or 11 (total $n=21$ ) MS patients, respectively $[27,33]$. Of note, the study by Beran and colleagues [27] used the ESS as a specific selection criterion (ESS score $\geq 9$ ) for patients with and without daytime sleepiness. In few other studies indicating pathological sleepiness on a group level, patients were selected regarding fatigue (fatigue descriptive scale $\geq 5$ ) in combination with complaints of sleep disturbances [58]. This was particularly the case for interventional studies investigating the efficacy of wakepromoting substances $[66,68]$. In some other studies showing increased ESS scores in patient subgroups, the patients had specifically been selected for increased fatigue levels [26,33,39,56].

In all studies with more than 150 MS patients, the mean ESS score did not indicate increased sleepiness. In the two largest samples of MS patients by Mills and Young [47] $(\mathrm{n}=559$ with valid ESS data) and Brass and colleagues [31] $(n=2375)$, the average ESS scores were 7.9 (mean) and 8 (median), respectively - well below the critical cut-off score. Despite the low mean ESS scores of the study sample published by Brass and colleagues [31], 30\% of the patients scored above 10 , indicating increased sleepiness. In general, daytime sleepiness does not seem to be a prominent and frequent symptom in MS patients on a group level, but may be present in a substantial number of patients (ranging up to $61 \%$ ) on an individual level (Table 2).

\section{The association of sleepiness and fatigue}

The majority of studies typically showed increased fatigue scores without overlapping sleepiness. Only one single study, comprising no more than 14 MS patients found increased ESS scores without corresponding elevated fatigue levels [60]. Our results indicate that fatigue without sleepiness is frequent, while sleepiness without fatigue is rare. Thus, if pathological fatigue is present, ESS scores can be either high or low. These findings are in accordance with a pilot study by Merkelbach and Schulz [22], indicating that sleepiness may vary to a noticeable extent independently from fatigue. The authors also suggest sleep disorders as a critical intervening factor that amplifies sleepiness in a subgroup of fatigued MS patients [46]. By extending their preliminary results in a larger study, the authors demonstrated in a single-item analysis of the ESS that only a subset of ESS items referring to self-paced activation for functioning was closely associated with fatigue as assessed by the FSS [46].

Few studies specifically distinguished between the concepts of sleepiness versus fatigue on a semantic level [27,46], while 19 studies investigated the relationship between both clinical conditions in more detail. These studies, which explored the relationship between the ESS as a main outcome parameter and fatigue scales found mainly moderate correlations between both conditions.

\section{Semantic confusion of concepts}

Sleepiness and fatigue are two interrelated but distinct phenomena [19] that can be easily confused [76]. The terms are often used as synonyms, both colloquially and in the scientific literature [77]. In clinical interviews, patients with hypersomnolence often use the term fatigue to describe their symptoms of excessive daytime sleepiness [78]. One particular study showed that patients with narcolepsy and patients with insomnia both scored high on the FSS when asked for symptoms of fatigue. However, 
only narcoleptic patients reported sleepiness as an increased propensity to fall asleep during the day assessed by the ESS [79]. In insomnia research, it is well established to differentiate between both concepts as clinical criteria, as patients with primary insomnia complain about excessive fatigue during the day, but not about sleepiness as defined above [80]. Because the meaning of both terms differs between languages, the assessment of fatigue and sleepiness is even more difficult. This is especially true for the FSS, which uses the term "fatigue" without any further specifications.

There is converging evidence that fatigue and sleepiness are two different concepts with a limited overlap of symptoms. Because fatigue is mostly poorly defined, applied assessment tools are often not precise enough to distinguish between both conditions [81]. Few original studies on MS (e.g., [27,46]) have explicitly distinguished between the concepts of sleepiness and fatigue and have explored the relationship between both clinical conditions in more detail. In many other studies, the terms fatigue and sleepiness are used inconsistently, sometimes even synonymously [76]. In general, the terms fatigue, tiredness and sleepiness are rarely explored differentially, and their lack of distinction is a major issue in clinical research and practice [21].

\section{The impact of sleep disorders}

Fatigue in MS appears to be multifactorial, with a component of fatigue directly attributable to the MS disease process (primary fatigue), as well as to secondary chronic illness factors, such as pain, medication, depression or other comorbidities, including poor sleep $[4,82,83]$. It has been reported that at least $50 \%$ of patients with MS complain about sleep disturbances or poor sleep $[5,84]$ ) Recent studies suggest that primary sleep disorders may intensify MS-related fatigue $[31,85]$. In addition, patients with MS are at an increased risk of secondary or comorbid forms of insomnia because common symptoms of MS (e.g., nocturia, pain, spasticity, paraesthesias, depression, and anxiety) often interfere with restorative sleep. Specific sleep disorders such as RLS, PLMD, and SRBD have also been reported to be present at higher frequencies in MS patients than in the general population $[4,6,33,86]$. Comorbid sleep disorders and poor sleep are often unrecognized clinical conditions in MS and may additionally contribute to fatigue [29,87]. This notion is supported by the findings that treatment of different sleep disorders, particularly SRBD, is effective to reduce fatigue in MS $[61,64,73]$.

The largest study included in our review comprised 2375 MS patients and focused on the frequency of sleep disorders and their association with fatigue and sleepiness [31]. As assessed by the ESS, the prevalence of daytime sleepiness was reported to be $30 \%$, while that for abnormal fatigue measures was $60 \%$. Increased sleepiness and fatigue scores were associated with positive screenings for insomnia, SRBD, and RLS that showed prevalence rates of $32 \%, 38 \%$, and $37 \%$, respectively. Thus, more than $70 \%$ of this large MS cohort screened positive for at least one or more sleep disorders [31]. Studies using sleep questionnaires, actigraphy or PSG found a positive correlation between subjectively or objectively measured disrupted sleep and fatigue in MS patients [26,27,40,48,56,61]. Kaynak and colleagues [41] found that the total arousal index was higher in a subgroup of fatigued patients compared to a non-fatigued group. However, another polysomnographic study investigating sleep-related correlates of fatigue in MS reported conflicting results [36]. In the case of increased daytime sleepiness, sleep disorders associated with hypersomnolence may be highly prevalent, yet under-recognized, clinical conditions in MS contributing to increased fatigue [29].

\section{Methodological constraints}

Our systematic review provides a qualitative and descriptive survey of the summarized studies that include original data. We may have failed to identify all relevant studies as the ESS might not be mentioned in the fields available via the databases' search interfaces. There was noticeable heterogeneity between studies with respect to study design, experimental procedures, and the quality of data sets on ESS or FSS. Therefore, no meta-analysis of effect sizes regarding sleepiness or fatigue scores was feasible for this review. As an operational definition of daytime sleepiness, we used sleep propensity as assessed by the ESS. However, the ESS is a subjective rating of sleep propensity in daily life and covers only one specific aspect of the multi-dimensional concept of sleepiness [88]. In addition, even though the ESS is widely used as an assessment tool in clinical practice and research, this instrument has only modest psychometric properties. The reliability and validity of the ESS as a unidimensional scale for sleepiness has been questioned by a number of studies [89]. However, due to the huge body of findings on the ESS and the knowledge of its strengths and limitations, utilizing the ESS as an assessment tool for evaluating sleepiness in MS seems to be an appropriate approach. Studies on MS comparing subjective and objective measures of sleepiness (e.g., multiple sleep latency test, maintenance of wakefulness test, or pupillography sleepiness test) are rare $[27,40,41,49,90]$ and beyond the scope of our review but warrant further research.

\section{Implications for clinical practice}

Sleepiness may confound measures developed to assess fatigue (see also Table 1). In general, individuals seem to subsume sleepiness under the broader notion of being tired or feeling fatigued. Thus, if patients with MS present fatigue as chief complaints or score high on fatigue scales, clinicians should be aware of the heterogeneity of the various symptoms. To evaluate sleepiness and fatigue in MS as two distinct clinical conditions, we suggest the following approach.

Firstly, MS patients with complaints of fatigue should be evaluated with regard to the severity and clinical significance of the symptoms (e.g., by using the FSS, the MFIS or other rating scales on the impact of fatigue). Secondly, sleepiness should be evaluated by the assessment of clinical signs of daytime sleepiness and supplemented by the use of the ESS. Increased daytime sleepiness may be indicative of sleep disorders associated with hypersomnolence and call for specific diagnostic procedures (ICSD-3). However, hypersomnolence can also be due to insufficient sleep caused by a lack of sleep hygiene or habitual short sleep times. Even if there are no signs of elevated sleep propensity during the day (i.e., ESS $\leq 10$ ), MS patients may complain about fatigue due to poor sleep or sleep disturbances, which are highly prevalent in MS [5,84]. Thus, overall fatigue or tiredness, but not sleepiness, is a core daytime symptom of insomnia that can perpetuate MS-related fatigue similarly to depression $[38,55,56]$. As mentioned above, sleep disturbances and poor sleep are common in MS and may contribute significantly to daytime fatigue.

Veauthier \& Paul suggested that MS patients with fatigue (e.g., MFIS >34) and impaired sleep quality (e.g., Pittsburgh sleep quality index (PSQI) > 5) should undergo further sleep evaluation and, if appropriate, sleep assessment in a sleep laboratory using PSG (e.g., to distinguish between pain, spasticity, and RLS) [6]. In cases of increased fatigue or sleepiness associated with non-restorative sleep, sleep disturbances observed by others (e.g., heavy snoring, apneas, leg movements) or impaired 
daytime functioning, MS patients should also be evaluated regarding sufficient sleep quantity and symptoms of specific sleep disorders such as RLS, PLMD, SRBD, or insomnia. Diagnosed sleep disorders have to be treated by sleep experts accordingly. The use of medications causing sedation or hypersomnia, as well as comorbid somatic or psychiatric disorders such as depression and anxiety, also need to be taken into account. Patients with symptomatic sleepiness may particularly benefit from wakefulness-promoting agents, activity, or daytime naps but not from rest [21]. If clinically significant fatigue is primary and intrinsic to the MS pathology, therapy should focus on fatigue-specific treatment such as energy conservation programs, cooling therapy or cognitive-behavioral therapy $[71,91,92]$.

\section{Conclusions}

Given the high but often underestimated prevalence of disturbed sleep in MS, patients with MS who suffer from fatigue that affects daily functioning should undergo a sleep evaluation if there are any signs of poor or non-restorative sleep. Within the prominent complaint of fatigue, sleepiness as a distinctive symptom can be easily overlooked. If patients with MS report fatigue or tiredness, specific aspects of unintentional sleep or the propensity to fall asleep should be assessed. In this context, the ESS, as a short and easy-to-administer questionnaire, may be used as an additional assessment tool. The presence of daytime sleepiness can guide diagnostic evaluations with respect to comorbid sleep disorders associated with hypersomnolence, allowing potential treatment by effective countermeasures of sleepiness (e.g., wakefulnesspromoting agents; avoidance of monotony, utilizing power naps, no rest).

Although sleepiness seems to be less prevalent and less severe than fatigue, it is present in a substantial number of patients with MS. Yet, sleepiness and tiredness-related fatigue due to sleep disturbances are both under-recognized and undertreated clinical conditions in MS that are in need of specific diagnoses and appropriate treatments.

\section{Practice points}

- Sleepiness and fatigue are two interrelated, albeit distinct concepts with limited overlap.

- In patients with MS, fatigue is more prevalent and severe than daytime sleepiness.

- Fatigue without sleepiness is frequent in MS, whereas sleepiness without fatigue is rare.

- High scores on fatigue scales are often accompanied by increased daytime sleepiness. Therefore, the ESS should also be applied in MS patients with increased fatigue scores. Both clinical conditions may be associated with sleep disorders.

- Increased sleepiness, as well as significant complaints of fatigue and poor sleep, warrants further assessment of potential comorbid sleep disorders or negative effects of hypnotic use. Elevated ESS scores may be indicative of sleep disorders associated with hypersomnolence and demand specific treatment of daytime sleepiness.

- The clinical significance of daytime sleepiness in MS patients remains unknown; nevertheless it may be presumed that it diminishes the quality of life of the patients.

\section{Research agenda}

- The assessment of the prevalence of daytime sleepiness in MS is needed. This requires a systematic epidemiological study using the ESS or other questionnaires in a large population of patients with MS focusing on the assessment of daytime sleepiness as the inability to stay awake and alert.

- Cross-sectional studies on distinct subtypes of MS should take into account both concepts of fatigue and sleepiness, using adequate and established assessment tools for each condition.

- Longitudinal studies are necessary to clarify whether the course of MS is related to the development of fatigue, sleepiness or sleep disorders.

- Prospective studies following MS patients with different subtypes should be performed to allow for the analysis of the association between fatigue, sleepiness or potential sleep disorders and the clinical MS status of the patients including the severity of disability as well as MRI findings.

- Studies comparing objective assessments of daytime sleepiness (e.g., maintenance of wakefulness test, pupillography, vigilance or sustained attention tasks) with subjective measures of sleepiness and fatigue are needed in patients with MS.

- In MS, systematic studies using the ESS or other screening tools for hypersomnolence are needed. Abnormal findings should be validated using more specific assessments (e.g., polysomnography).

\section{Conflicts of interest}

The authors have no conflict of interest to disclose.

\section{Appendix A. Supplementary data}

Supplementary data related to this article can be found at http:// dx.doi.org/10.1016/j.smrv.2016.03.004.

\section{References}

[1] Weissert R. The immune pathogenesis of multiple sclerosis. J Neuroimmune Pharmacol 2013:8:857-66.

[2] Browne P, Chandraratna D, Angood C, Tremlett H, Baker C, Taylor BV, et al Atlas of multiple sclerosis 2013: a growing global problem with widespread inequity. Neurology 2014;83:1022-4.

[3] Lublin FD, Reingold SC, Cohen JA, Cutter GR, Sørensen PS, Thompson AJ, et al. Defining the clinical course of multiple sclerosis: the 2013 revisions. Neurology 2014:83:278-86.

*[4] Krupp LB, Serafin DJ, Christodoulou C. Multiple sclerosis-associated fatigue. Expert Rev Neurothera 2010;10:1437-47.

[5] Merlino G, Fratticci L, Lenchig C, Valente M, Cargnelutti D, Picello M, et al. Prevalence of 'poor sleep' among patients with multiple sclerosis: an independent predictor of mental and physical status. Sleep Med 2009;10(1): 26-34.

[6] Veauthier C, Paul F. Sleep disorders in multiple sclerosis and their relationship to fatigue. Sleep Med 2014;15(1):5-14.

[7] Asenbaum S, Zeitlhofer J, Kollegger H, Klösch G, Vass K. Sleep quality and fatigue in multiple sclerosis- preliminary results. Sleep Res Online 1999;2(Suppl. 1):475.

[8] Colosimo C, Millefiorini E, Grasso MG, Vinci F, Fiorelli M, Koudriavtseva T, et al. Fatigue in MS is associated with specific clinical features. Acta Neurol Scand 1995;92(5):353-5.

\footnotetext{
* The most important references are denoted by an asterisk.
} 
[9] Iriarte J, Subirá ML, Castro P. Modalities of fatigue in multiple sclerosis: correlation with clinical and biological factors. Mult Scler 2000;6(2): 124-30.

[10] Krupp LB, Pollina DA. Mechanisms and management of fatigue in progressive neurological disorders. Curr Opin Neurol 1996;9(6):456-60.

[11] Vercoulen JH, Hommes OR, Swanink CM, Jongen PJ, Fennis JF, Galama JM, et al. The measurement of fatigue in patients with multiple sclerosis. A multidimensional comparison with patients with chronic fatigue syndrome and healthy subjects. Arch Neurol 1996;53(7):642-9.

[12] Freal JE, Kraft GH, Coryell JK. Symptomatic fatigue in multiple sclerosis. Arch Phys Med Rehabil 1984;65(3):135-8.

[13] Krupp LB, LaRocca NG, Muir-Nash J, Steinberg AD. The fatigue severity scale. Application to patients with multiple sclerosis and systemic lupus erythematosus. Arch Neurol 1989;46(10):1121-3.

[14] Krupp LB, La Alvarez, LaRocca NG, Scheinberg LC. Fatigue in multiple sclerosis. Arch Neurol 1988;45(4):435-7.

[15] Kinkel RP. Fatigue in multiple sclerosis: reducing the impact through comprehensive management. Int J MS Care 2000;2(3):3-10.

[16] Hadjimichael O, Vollmer T, Oleen-Burkey M. Fatigue characteristics in multiple sclerosis: the North American Research Committee on Multiple Sclerosis (NARCOMS) survey. Health Qual Life Out 2008;6:100.

[17] Smith MM, Arnett PA. Factors related to employment status changes in individuals with multiple sclerosis. Mult Scler 2005;11(5):602-9.

[18] American Academy of Sleep Medicine. International classification of sleep disorders. 3rd ed. IL, USA: Darien; 2014.

[19] Shen J, Barbera J, Shapiro CM. Distinguishing sleepiness and fatigue: focus on definition and measurement. Sleep Med Rev 2006;10(1):63-76.

[20] Sukovic A. Measuring the meaning of "sleepy", "fatigued" and "tired": a semantic differential study [Thesis]. Flinders University; 2007.

[21] Neu D, Linkowski P, le Bon O. Clinical complaints of daytime sleepiness and fatigue: how to distinguish and treat them, especially when they become ‘excessive' or 'chronic'? Acta Neurol Belg 2010;110(1):15-25.

[22] Merkelbach S, Schulz $\mathrm{H}$. What have fatigue and sleepiness in common? J Sleep Res 2006;15(1):105-6.

*[23] Johns MW. A new method for measuring daytime sleepiness: the Epworth sleepiness scale. Sleep 1991;14(6):540-5.

[24] McGowan J, Sampson M, Lefebvre C. An evidence based checklist for the pee review of electronic search strategies (PRESS EBC). Evid Based Libr Inf Pract 2010;5(1):149-54.

[25] Atkinson KM, Koenka AC, Sanchez CE, Moshontz H, Cooper H. Reporting standards for literature searches and report inclusion criteria: making research syntheses more transparent and easy to replicate. Res Syn Meth 2015;6(1):87-95.

*[26] Attarian HP, Brown KM, Duntley SP, Carter JD, Cross AH. The relationship of sleep disturbances and fatigue in multiple sclerosis. Arch Neurol 2004;61(4): 525-8.

[27] Beran RG, Ainley LA, Holland G. Sleepiness in multiple sclerosis: a pilot study. Sleep Biol Rhythms 2008;6(4):194-200.

*[28] Braley TJ, Chervin RD, Segal BM. Fatigue, tiredness, lack of energy, and sleepiness in multiple sclerosis patients referred for clinical polysomnography. Mult Scler Int 2012;2012:673936.

[29] Braley T, Segal B, Chervin RD. Obstructive sleep apnea and fatigue in patients with multiple sclerosis. J Clin Sleep Med 2014;10(2):155-62.

[30] Braley TJ, Segal BM, Chervin RD. Hypnotic use and fatigue in multiple sclerosis. Sleep Med 2015;16(1):131-7.

*[31] Brass SD, Li CS, Auerbach S. The underdiagnosis of sleep disorders in patients with multiple sclerosis. J Clin Sleep Med 2014;10(9):1025-31.

*[32] Bøe Lunde HM, Aae TF, Indrevåg W, Aarseth J, Bjorvatn B, Myhr KM, et al Poor sleep in patients with multiple sclerosis. PLoS One 2012;7(11):e49996.

[33] Chen JH, Liu XQ, Sun HY, Huang Y. Sleep disorders in multiple sclerosis in China: clinical, polysomnography study, and review of the literature. J Clin Neurophysiol 2014;31(4):375-81.

[34] Constantinescu CS, Niepel G, Patterson M, Judd A, Braitch M, Orexin A (hypocretin-1) levels are not reduced while cocaine/amphetamine regulated transcript levels are increased in the cerebrospinal fluid of patients with multiple sclerosis: no correlation with fatigue and sleepiness. J Neurol Sci 2011;307(1):127-31.

[35] Dias RA, Hardin KA, Rose H, Agius MA, Apperson ML, Brass SD. Sleepiness fatigue, and risk of obstructive sleep apnea using the STOP-BANG questionnaire in multiple sclerosis: a pilot study. Sleep Breath 2012;16(4):1255-65.

[36] Elkattan MM, El-Serafy OA, Helmy SM, Kishk NA. Exploring the relationship between fatigue and sleep disturbances in multiple sclerosis. Egypt J Neurol Psychiat Neurosurg 2009;46(1):193-202.

[37] Frauscher B, Egg R, Brandauer E, Ulmer H, Berger T, Poewe W, et al. Daytime sleepiness is not increased in mild to moderate multiple sclerosis: a pupil lographic study. Sleep Med 2005;6(6):543-7.

[38] Ghajarzadeh M, Sahraian MA, Fateh R, Daneshmand A. Fatigue, depression and sleep disturbances in Iranian patients with multiple sclerosis. Acta Med Iran 2012:50(4):244-9.

[39] Heesen C, Nawrath L, Reich C, Bauer N, Schulz K, Gold SM. Fatigue in multiple sclerosis: an example of cytokine mediated sickness behaviour? J Neurol Neurosurg Psychiatry 2006;77(1):34-9.

[40] Kaminska M, Kimoff RJ, Benedetti A, Robinson A, Bar-Or A, Lapierre Y, et al Obstructive sleep apnea is associated with fatigue in multiple sclerosis. Mult Scler 2012;18(8):1159-69.
[41] Kaynak H, Altintaş A, Kaynak D, Uyanik O, Saip S, Ağaoğlu J, et al. Fatigue and sleep disturbance in multiple sclerosis. Eur J Neurol 2006;13(12):1333-9.

[42] Kister I, Caminero AB, Monteith TS, Soliman A, Bacon TE, Bacon JH, et al Migraine is comorbid with multiple sclerosis and associated with a more symptomatic MS course. J Headache Pain 2010;11(5):417-25.

[43] Knudsen S, Jennum PJ, Korsholm K, Sheikh SP, Gammeltoft S, Frederiksen JL. Normal levels of cerebrospinal fluid hypocretin-1 and daytime sleepiness during attacks of relapsing-remitting multiple sclerosis and monosymptomatic optic neuritis. Mult Scler 2008;14(6):734-8.

[44] Kotterba S, Eren E, Fangerau T, Malin J, Sindern E. Sleepiness and fatigue in multiple sclerosis - comparison of different measuring instruments. Fortschr Neurol Psychiatr 2003;71(11):590-4.

[45] Labuz-Roszak B, Kubicka-Baczyk K, Pierzchala K, Machowska-Majchrzak A, Skrzypek M. Fatigue and its association with sleep disorders, depressive symptoms and anxiety in patients with multiple sclerosis. Neurol Neurochir Pol 2012;46(4):309-17.

*[46] Merkelbach S, Schulz H, Kölmel HW, Gora G, Klingelhöfer J, Dachsel R, et al. Fatigue, sleepiness, and physical activity in patients with multiple sclerosis. J Neurol 2011;258(1):74-9.

[47] Mills RJ, Young CA. The relationship between fatigue and other clinical features of multiple sclerosis. Mult Scler 2011;17(5):604-12.

[48] Moreira NC, Damasceno RS, Medeiros CA, Bruin PF, Teixeira CA, Horta WG, et al. Restless leg syndrome, sleep quality and fatigue in multiple sclerosis patients. Braz J Med Biol Res 2008;41(10):932-7.

*[49] Neau J, Paquereau J, Auche V, Mathis S, Godeneche G, Ciron J, et al. Sleep disorders and multiple sclerosis: a clinical and polysomnography study. Eur Neurol 2012;68(1):8-15.

[50] Neumann M, Sterr A, Claros-Salinas D, Gütler R, Ulrich R, Dettmers C. Modulation of alertness by sustained cognitive demand in MS as surrogate measure of fatigue and fatigability. J Neurol Sci 2014;340(1-2):178-82.

[51] Niepel G, Bibani RH, Vilisaar J, Langley RW, Bradshawb CM, Szabadi E, et al. Association of a deficit of arousal with fatigue in multiple sclerosis: effect of modafinil. Neuropharmacology 2013;64:380-8.

[52] Papuć E, Stelmasiak Z, Grieb P, Rejdak K. CSF hypocretin-1 concentrations correlate with the level of fatigue in multiple sclerosis patients. Neurosci Lett 2010;474(1):9-12.

[53] Pokryszko-Dragan A, Bilińska M, Gruszka E, Biel Ł, Kamińska K, Konieczna K. Sleep disturbances in patients with multiple sclerosis. Neurol Sci 2013;34(8): 1291-6.

[54] Sauter MC. Abnorme Tagesmüdigkeit bei Multipler Sklerose: Zusammenhänge mit Fatigue, Tagesschläfrigkeit, Vigilanz, Depression, Angst und Lebensqualität [Dissertation]. Wien: Universität Wien; 2004.

[55] Stanton BR, Barnes F, Silber E. Sleep and fatigue in multiple sclerosis. Mult Scler 2006:12(4):481-6.

*[56] Veauthier C, Radbruch H, Gaede G, Pfueller CF, Dörr J, Bellmann-Strobl J, et al. Fatigue in multiple sclerosis is closely related to sleep disorders: a polysomnographic cross-sectional study. Mult Scler 2011;17(5):613-22.

[57] Wunderlin BW, Kesselring J, Ginzler H, Walser B, Kuhn M, Reinhart WH Fatigue in multiple sclerosis is not due to sleep apnoea. Eur J Neurol $1997 ; 4(1): 72-8$.

[58] Attarian H, Applebee G, Applebee A, Wang B, Clark M, McCormick, et al. Effect of eszopiclone on sleep disturbances and daytime fatigue in multiple sclerosis patients. Int J MS Care 2011;13(2):84-90.

[59] Brioschi A, Graminga S, Werth E, Staub F, Ruffieux C, Bassetti C, et al. Effect of modafinil on subjective fatigue in multiple sclerosis and stroke patients. Eur Neurol 2009;62(4):243-9.

[60] Bruce J, Hancock L, Roberg B, Brown A, Henkelman E, Lynch S. Impact of armodafinil on cognition in multiple sclerosis: a randomized, double-blind crossover pilot study. Cogn Behav Neurol 2012;25(3):107-14.

[61] Côté I, Trojan DA, Kaminska M, Cardoso M, Benedetti A, Weiss D, et al. Impact of sleep disorder treatment on fatigue in multiple sclerosis. Mult Scler 2013;19(4):480-9.

[62] García Jalón EG, Lennon S, Peoples L, Murphy S, Lowe-Strong A. Energy conservation for fatigue management in multiple sclerosis: a pilot randomized controlled trial. Clin Rehabil 2013;27(1):63-74.

[63] Gerhard R. Fatigue und Tagesschläfrigkeit bei Patienten mit schubförmig remittierender Multipler Sklerose (RR-MS) unter Therapie mit Interferon beta-1a (Avonex) [Dissertation]. Bochum: Ruhr-Universität Bochum; 2009.

[64] Kallweit U, Baumann CR, Harzheim M, Hidalgo H, Pöhlau D, Bassetti CL, et al. Fatigue and sleep-disordered breathing in multiple sclerosis: a clinically relevant association. Mult Scler Int 2013;2013:1-7.

[65] Mendozzi L, Tronci F, Garegnani M, Pugnetti L. Sleep disturbance and fatigue in mild relapsing remitting multiple sclerosis patients on chronic immunomodulant therapy;an actigraphic study. Mult Scler 2010;16(2):238-47.

[66] Möller F, Poettgen J, Broemel F, Neuhaus A, Daumer M, Heesen C. HAGIL (Hamburg Vigil Study): a randomized placebo-controlled double-blind study with modafinil for treatment of fatigue in patients with multiple sclerosis. Mult Scler 2011;17(8):1002-9.

[67] Rammohan KW, Rosenberg JH, Lynn DJ, Blumenfeld Am, Pollak CP, Nagaraja HN. Efficacy and safety of modafinil (Provigil) for the treatment of fatigue in multiple sclerosis: a two centre phase 2 study. J Neurol Neurosurg Psychiatry 2002;72(2):179-83.

[68] Stankoff B, Waubant E, Confavreux E, Edan G, Debouverie M. Modafinil for fatigue in MS: a randomized placebo-controlled double-blind study. Neurology 2005;64(7):1139-43. 
[69] Svenningsson A, Falk E, Celius EG, Fuchs F, Schreiber K, Berkö S, et al. Netalizumab treatment reduces fatigue in multiple sclerosis. Results from the TYNERGY Trial;A study in the real life setting. PLoS One 2013;8(3):e58643.

[70] Vakhapova V, Auriel E, Karni A. Nightly sublingual tizanidine $\mathrm{HCl}$ in multiple sclerosis: clinical efficacy and safety. Clin Neuropharmacol 2010;33(3):151-4.

[71] van Kessel K, Moss-Morris R, Willoughby E, Chalder T, Johnson MH, Robinson E. A randomized controlled trial of cognitive behavior therapy for multiple sclerosis fatigue. Psychosom Med 2008;70(2):205-13.

[72] Zifko UA, Rupp M, Schwarz S, Zipko HT, Maida EM. Modafinil in treatment of fatigue in multiple sclerosis. J Neurol 2002;249(8):983-7.

[73] Veauthier C, Gaede G, Radbruch H, Gottschalk S, Wernecke K, Paul F. Treatment of sleep disorders may improve fatigue in multiple sclerosis. Clin Neurol Neurosurg 2013;115(9):1826-30.

[74] Kaminska M. Sleep-disordered breathing and fatigue in multiple sclerosis [Thesis]. McGill University Library; 2010.

[75] Mücke M, Mochamat, Cuhls H, Peuckmann-Post V, Minton O, Stone P, et al. Pharmacological treatments for fatigue associated with palliative care. Cochrane Database Syst Rev 2015:5.

[76] Hossain JL, Ahmad P, Reinish LW, Kayumov L, Hossain NK, Shapiro CM. Subjective fatigue and subjective sleepiness: two independent consequences of sleep disorders? J Sleep Research 2005;14(3):245-53.

[77] Sukovic A, Lack LC, Wright HR. Quantifying the differences in meaning between "sleepy", “tired", and "fatigued". J Sleep Research 2008;17(Suppl. 1): 176.

[78] Chervin RD. Sleepiness, fatigue, tiredness, and lack of energy in obstructive sleep apnea. Chest 2000;118(2):372-9.

[79] Valko PO, Bassetti CL, Bloch KE, Held U, Baumann CR. Validation of the fatigue severity scale in a Swiss cohort. Sleep 2008;31(11):1601-7.

[80] Buysse DJ, Ancoli-Israel S, Edinger JD, Lichstein KL, Morin CM. Recommendations for a standard research assessment of insomnia. Sleep 2006;29(9): $1155-73$.
[81] Pigeon WR, Sateia MJ, Ferguson RJ. Distinguishing between excessive daytime sleepiness and fatigue: toward improved detection and treatment. J Psychosom Res 2003;54(1):61-9.

[82] Multiple Sclerosis Council for Clinical Practice Guidelines. Fatigue and multiple sclerosis: evidence-based management strategies for fatigue in multiple sclerosis. Washington, DC: Paralyzed Veterans of America; 1998.

[83] Kos D, Kerckhofs E, Nagels G, D'hooghe MB, Ilsbroukx S. Origin of fatigue in multiple sclerosis: review of the literature. Neurorehabil Neural Repair 2008:22(1):91-100.

[84] Tachibana N, Howard RS, Hirsch NP, Miller DH, Moseley IF, Fish D. Sleep problems in multiple sclerosis. Eur Neurol 1994;34(6):320-3.

[85] Kaminska M, Kimoff RJ, Schwartzman K, Trojan DA. Sleep disorders and fatigue in multiple sclerosis: evidence for association and interaction. J Neurol Sci 2011;302(1-2):7-13.

[86] Sieminski M, Losy J, Partinen M. Restless legs syndrome in multiple sclerosis. Sleep Med Rev 2015;22:15-22.

[87] Brass S, Duquette P, Proulx-Therrien J, Auerbach S. Sleep disorders in patients with multiple sclerosis. Sleep Med Rev 2010;14(2):121-9.

[88] Cluydts R, Valck E, Verstraeten E, Theys P. Daytime sleepiness and its evaluation. Sleep Med Rev 2002;6(2):83-96.

*[89] Kendzerska TB, Smith PM, Brignardello-Petersen R, Leung RS, Tomlinson GA. Evaluation of the measurement properties of the Epworth sleepiness scale: a systematic review. Sleep Med Rev 2014;18(4):321-31.

[90] Egg R, Högl B, Glatzl S, Beer R, Berger T. Autonomic instability, as measured by pupillary unrest, is not associated with multiple sclerosis fatigue severity. Mult Scler 2002;8(3):256-60.

[91] Sauter C, Zebenholzer K, Hisakawa J, Zeitlhofer J, Vass K. A longitudinal study on effects of a six-week course for energy conservation for multiple sclerosis patients. Mult Scler 2008;14(4):500-5.

[92] Schwid SR, Petrie MD, Murray R, Leitch J, Bowen J, Alquist A, et al A randomized controlled study of the acute and chronic effects of cooling therapy for MS. Neurology 2003;60:1955-60. 